\title{
The role of Fnr paralogs for controlling anaerobic metabolism in
}

\section{diazotroph Paenibacillus polymyxa WLY78}

Haowen Shi, Yongbin Li, Tianyi Hao, Xiaomeng Liu, Xiyun Zhao, Sanfeng Chen"

State Key Laboratory for Agrobiotechnology, College of Biological Sciences and Key Laboratory

of Soil Microbiology of Agriculture Ministry, China Agricultural University, Beijing, P. R. China

Running title: Role of Fnr paralogs in Paenibacillus polymyxa WLY78

\#Address correspondence to Sanfeng Chen, chensf@cau.edu.cn

Haowen Shi: shw_126@126.com

Yongbin Li: 280217065@qq.com

Tianyi Hao: sz20143020027@cau.edu.cn

Xiaomeng Liu: wsw_lxm2012@163.com

Xiyun Zhao: zhaoxy08@163.com

Keywords: Fnr, Paenibacillus polymyxa, anaerobic regulation, biological nitrogen fixation

(1)

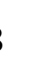

9

0

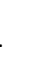

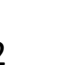

3

24

5

6




\section{ABSTRACT}

Fnr is a transcriptional regulator that controls the expression of a variety of genes in response to oxygen limitation in bacteria. Genome sequencing revealed four genes ( $f n r l, f n r 3, f n r 5$ and $f n r 7)$ coding for Fnr proteins in Paenibacillus polymyxa WLY78. Fnr1 and Fnr3 showed more similarity to each other than to Fnr5 and Fnr7. Also, Fnr1 and Fnr3 exhibited high similarity with Bacillus cereus Fnr and Bacillus subtilis Fnr in sequence and structures. Deletion analysis showed that the four $f n r$ genes, especially $f n r l$ and $f n r 3$, have significant impacts on the growth and nitrogenase activity. Single deletion of fnrl or fnr3 led to $50 \%$ reduction in nitrogenase activity and double deletion of fnrl and fnr3 resulted to $90 \%$ reduction in activity. Both of the aerobically purified His-tagged Fnr1 and His-tagged Fnr3 in Escherichia coli could bind to the specific DNA promoter. Genome-wide transcription analysis showed that Fnr1 and Fnr3 indirectly activated expression of nif (nitrogen fixation) genes and Fe transport genes under anaerobic condition. Fnr1 and Fnr3 inhibited expression of the genes involved in aerobic respiratory chain and activated expression of genes responsible for anaerobic electron acceptor genes.

\section{IMPORTANCE}

Paenibacillus is a genus of Gram-positive, facultative anaerobic and endospore-forming bacteria. The members of nitrogen-fixing Paenibacillus have great potential use as a bacterial fertilizer in agriculture. However, the functions of $f n r$ gene(s) in nitrogen fixation and other metabolisms in Paenibacillus spp. are not known. Here, we revealed that copy numbers vary largely among different Paenibacillus species and strains. Deletion and complementation analysis demonstrated that fnrl and fnr3 have significant impacts on the growth and nitrogenase activity. Both of the aerobically purified His-tagged Fnr1 and His-tagged Fnr3 purified in Escherichia coli could bind to the specific DNA promoter as Bacillus cereus Fnr did. Fnr1 and Fnr3 indirectly activated nif expression under anaerobic condition. Fnr1 and Fnr3 directly or indirectly activated or inhibited expression of many important genes involved in respiration, energy metabolism, Fe uptake and potentially specific electron transport for nitrogenase under anaerobic condition. This study not only reveals the roles of $f n r$ genes in nitrogen fixation and anaerobic metabolism, but also provides insight into the evolution and regulatory mechanisms of fnr in Paenibacillus. 


\section{INTRODUCTION}

Most biological nitrogen fixation is catalyzed by molybdenum-dependent nitrogenase, which is distributed within bacteria and archaea. This enzyme is composed of two metalloproteins: MoFe protein and Fe protein (1). Nitrogenase is an oxygen-sensitive enzyme, and both the $\mathrm{MoFe}$ and $\mathrm{Fe}$ proteins are irreversibly damaged by oxygen (2). $\mathrm{O}_{2}$ exposure leads to inappropriate oxidation of the metalloclusters, decrease of protein secondary structure and further degradation (3). Exposure to oxygen irreversibly inactivates the Mo-, V-, and Fe-nitrogenases (3-5). To avoid oxygen inactivation, diazotrophs (nitrogen-fixing organisms) have evolved different strategies. One of the strategies is to tightly control the transcription of nitrogen fixation genes (nif) in response to the external oxygen concentration.

Fnr (fumarate and nitrate reduction) protein is a global regulator that binds a $[4 \mathrm{Fe}-4 \mathrm{~S}]$ cluster to monitor the oxygen status in the cell and then controls transcription of lot of genes in response to changes in oxygen levels (6-9). Fnr is widely distributed in Gram-negative bacteria (e.g. Escherichia coli) (10) and Gram-positive bacteria (e.g. Bacillus subtilis) (11). Fnr-related transcriptional regulators of the Crp/Fnr (cyclic AMP-binding protein/fumarate nitrate reduction regulatory protein) family have been reported to be involved in nitrogen fixation of some Gram-negative diazotrophs (12-15). For example, Fnr proteins are indirectly involved in controlling the activity of NifA in Herbaspirillum seropedicae SmR1 by regulating respiratory activity in relation to oxygen availability $(16,17)$. Fnr protein of Klebsiella oxytoca is required to relieve inhibition of NifA activity by its partner regulatory protein NifL under anaerobic conditions (14). In symbiotic Bradyrhizobium japonicum and Sinorhizobium meliloti, transcription of nifA and fix genes is predominantly controlled by the oxygen-responsive two component FixL-FixJ system, together with FixK which is a member of the Crp/Fnr superfamily, or by the redox-sensing system RegS-RegR (12,13). In Rhizobium leguminosarium UPM791 FnrN is responsible for the expression of the high affinity oxidase encoded by fixNOQP which supports growth under microaerobic conditions and is essential for nitrogen fixation (15).

Paenibacillus polymyxa WLY78 can fix nitrogen under anaerobic or microaerobic and nitrogen-limited conditions and has a nif operon composed of 9 genes (nifBHDKENXhesAnifV)

under the control of a $\sigma^{70}$-dependent promoter in front of nifB gene (18). Recently, we have revealed that GlnR of $P$. polymyxa WLY78 activates nif transcription under anaerobic and 
nitrogen-limited condition, but GlnR together with glutamine synthetase (GS, glnA product) represses nif transcription under excess nitrogen and anaerobic condition (19).

Here, we searched the genome of $P$. polymyxa WLY78 and found that there are four genes coding for Fnr proteins. A total of $12 \mathrm{fnr}$ deletion mutants, including single, double, triple and quadruple $f n r$ deletion mutants were constructed by homologous recombination. The growth rates and nitrogenase activities among these $f n r$ mutants and wild-type P. polymyxa WLY78 were comparatively analyzed. Each of the single deletion mutants $\Delta f n r l, \Delta f n r 3, \Delta f n r 5$ and $\Delta f n r 7$ was effectively complemented by its corresponding fnr gene and by B. subtilis fnr. His-tagged Fnr1 and His-tagged Fur3 proteins expressed and purified in E. coli under aerobic conditions were used to verify the target genes by EMSA. Genome-wide transcription analysis in P. polymyxa WLY78 and the double mutant $\Delta f n r 13$ were performed.

\section{RERULTS}

Identification of $\boldsymbol{f n r}$ genes in P. polymyxa. Analysis of the P. polymyxa WLY78 genome showed four fnr-like genes (named as fnrl (S6001676), fnr3 (S6003218), fnr5 (S6004820) and fnr7 (S6005182)) (18). There are 39.98-53.63\% identity among the four Fnr1, Fnr3, Fnr5 and Fnr7 proteins of P. polymyxa at amino acid level (Table S1). The highest (53.63\%) identity was found between Fnr1 and Fnr3. Fnr1 and Fnr3 are more similar to each other than to Fnr5 and Fnr7. Like P. polymyxa WLY78, the three strains P. polymyxa M1, P. polymyxa E681, and P. polymyxa SC2 have four $f n r$ genes. Each of the four $f n r$ genes shows $99.44-100 \%$ identity with its corresponding gene from the different $P$. polymyxa strains (Table S1). However, some Paenibacillus species or strains, such as Paenibacillus polymyxa EBL06, Paenibacillus polymyxa Sb3-1 and Paenibacillus jamilae NS115 have only one Fnr which has 16.34-34.78\% identity with the four Fnr proteins of $P$. polymyxa WLY78. Also, Fnr1, Fnr3, Fnr5 and Fnr7 proteins of P. polymyxa share 50.94\%, 52.99\%, 45.45\% and $43.19 \%$ identities with of B. subtilis Fnr protein, respectively. Whereas, Fnr1 and Fnr3. Also, Fnr1, Fnr3, Fnr5 and Fnr7 proteins of P. polymyxa have 41.57\%, 45.08\%, 19.01\% and $21.82 \%$ identities with Bacillus cereus Fnr.

The four Fnr proteins of $P$. polymyxa WLY78 contain the predicted N-terminal receiver domain and C-terminal DNA-binding domain (Fig. 1A), which represents the feature of the Crp/Fnr family protein (7). The $[4 \mathrm{Fe}-4 \mathrm{~S}]{ }^{2+}$ cluster of B. subtilis Fnr is coordinated by three 
C-terminally located cysteine residues at positions 227, 230, and 235 and one aspartate residue at position 141 (7, 20). Similar to B. subtilis Fnr, Fnr1 and Fnr3 proteins of P. polymyxa WLY78 have these conserved cysteine and aspartate residues. But Fnr5 and Fnr7 proteins of P. polymyxa WLY78 lack these conserved residues (Fig. 1A). The data suggest that Fnr1 and Fnr3 proteins of $P$. polymyxa WLY78 show high similarity with $B$. subtilis Fnr and B. cereus Fnr in sequence and structure.

A phylogenetic analysis showed that the four $P$. polymyxa Fnr proteins followed into 3 groups (Fig. 1B). Fnr1 and Fnr3 are in the clade with the Fnr group of Bacillaceae. Fnr5 is near the clade with the Fnr group from Listeria and FixK group of Sporanaerobacter and Clostridiales, while Fnr7 is divergent from Fnr and FixK group of Bacillaceae. The data are consistent with the protein homology analysis.

Influence of $f$ r on growth under anaerobic condition. To explore the regulatory function of the four Fnr proteins of $P$. polymyxa WLY78, 12 unmarked $f n r$ deletion $(\Delta f n r)$ mutants, including single, double, triple and quadruple deletion mutants, were constructed as described in the Methods. The number in the $\Delta f n r$ mutant indicates which $f n r$ gene is deleted (e.g. $\Delta f n r l$ indicates deleting fnrl gene, $\Delta f n r 13$ indicating deleting both $f n r l$ and $f n r 3$ genes).

As Fnr protein is known to sense oxygen and plays a major role in altering gene expression polymyxa WLY78 under anaerobic conditions is here investigated. P. polymyxa WLY78 and multiple fnr deletion mutants were cultivated in nitrogen deficient medium with casamino acid under anaerobic and aerobic conditions (Fig. 2A). Except for the double fnr deletion mutant $\Delta f n r 57$, all of the $f n r$ deletion mutants showed lower growth rate than $P$. polymyxa WLY78 did. Compared to wild-type $P$. polymyxa WLY78, each single $f n r$ deletion mutant showed slow growth rate. The quadruple $f n r$ deletion mutant $\Delta f n r 1357$ showed the lowest growth rates among all of the $12 \Delta f n r$ mutants, suggesting that the four $f n r$ genes play roles under anaerobic condition. Notably, the single deletion mutants $\Delta f n r l$ and $\Delta f n r 3$ and the double $f n r$ deletion mutant $\Delta f n r l 3$ showed very low growth rate, suggesting that Fnr1 and Fnr3 proteins play an important role in anaerobic metabolisms in response to oxygen.

Effects of f $\boldsymbol{n} \boldsymbol{r}$ on nitrogenase activity. Since nitrogenase is very sensitive to $\mathrm{O}_{2}$, nitrogen fixation is performed in anaerobic or microanaerobic conditions. To determine if Fnr proteins are related to 
nitrogen fixation, the nitrogenase activities of wide-type P. polymyxa WLY78 and multiple fnr deletion mutants grown anaerobically in nitrogen deficient medium were measured by using the method of the reduction of acetylene to ethylene $(21,22)$. As shown in Fig. 2B, the nitrogenase activities of $\Delta f n r l$ and $\Delta f n r 3$ were decreased to about $50 \%$ of the wild type, while the activities of $\Delta f n r 5$ and $\Delta f n r 7$ were decreased to about $73-79 \%$ of the wild type. And the nitrogenase activity of $\Delta f n r 37$ and $\Delta f n r 13$ were decreased to about $36 \%$ and $10 \%$ of the wild type, respectively. Notably, the nitrogenase activities of $\Delta f n r l 37$ and $\Delta f n r 1357$ were nearly lost. The data are consistent with the growth rates of these mutants observed as above. The results imply that the four fnr genes, especially fnrl and fnr3, play roles in nitrogen fixation.

Furthermore, complementation of $\Delta f n r 1, \Delta f n r 3, \Delta f n r 5$ and $\Delta f n r 7$ with its corresponding $P$. polymyxa fnr gene and B. subtilis fnr gene under the control of its own promoter was performed. As shown in Fig. S1, fnrl, fnr3, fnr5 and fnr7 from P. polymyxa WLY78 in complemented strains $(\Delta f n r l C, \triangle f n r 3 C, \Delta f n r 5 C$ and $\triangle f n r 7 C$ ) restored the nitrogenase activity of its corresponding mutant to more than $90 \%$ activity of wild type. Complementation with His-tagged Fnr1 and His-tagged Fnr3 in complemented strains ( $\triangle$ fnrlChis and $\triangle$ fnr3CHis) also restored the nitrogenase activity of its corresponding mutant to more than $90 \%$ activity of the wild type. Moreover, we found that B. subtilis fnr gene greatly improved the nitrogenase activity of the four single $f n r$ deletion mutants, especially the activities of $\Delta f n r l$ and $\Delta f n r 3$. Also, B. subtilis fnr gene greatly restored the nitrogenase activities of the multiple deletion mutants $\Delta$ fnrl3, $\Delta$ fnrl37 and $\Delta$ fnrl357. The data confirm that the four fnr genes of P. polymyxa WLY78, especially fnrl and fnr3, play an important role in nitrogen fixation. The data suggest that Fnr1 and Fnr3 of $P$. polymyxa and Fnr of B. subtilis are similar in function.

The nifH transcription in the $f n r$ deletion mutants. The $n i f H$ transcriptions in different mutants were assayed by qRT-PCR (Fig. S2A). The nifH in $\Delta f n r 3$ was expressed at basic level. The nifH transcriptions in $\Delta f n r l, \Delta f n r 5$ and $\Delta f n r 7$ were decreased to about $40 \%, 70 \%$ and $90 \%$ of wild type, respectively. Whereas, the nifH transcriptions in $\Delta f n r l 3$ and $\Delta f n r 1357$ were nearly lost. Furthermore, the effects of $f n r$ on nif expression were performed by measuring the $\beta$-galactosidase activity of $P$. polymyxa WLY78 and fnr deletion mutants that carrying a transcriptional lacZ fusion 
activities and the nifH transcriptions in these fnr mutants.

Prediction and verification of Fnr target genes. To decipher the Fnr regulon of P. polymyxa WLY78, its target genes were predicted. According to the known Fnr-binding sequence of Bacillus and Paenibacillus in RegPrecise (http://regprecise.lbl.gov), the PWM (position weight matrix) of Fnr-binding site was constructed using MEME (http://meme-suite.org). The Fnr-binding consensus motif composed of a 16 bp palindromic sequence 5'-TGTGA-N6-TCACA-3' was determined. Then we used the $16 \mathrm{bp}$ Fnr consensus binding-motif to scan the regions from -350 to $+50 \mathrm{bp}$ relative to the translational start codon (ATG) of genes in P. polymyxa WLY78 genome with the MAST application (http://meme-suite.org) (23). A total of 143 putative Fnr target genes with the E-value $\leq 10$ (the smaller the E-value, the greater the probability) form $P$. polymyxa WLY78 genome were identified (Table 1). As annotated by the COG (Cluster of Orthologous Group), the 143 putative target genes were allocated to 12 groups by biological function. Of the 143 putative target genes, 19 belong to regulatory genes, 23 genes are related to energy metabolism, 11 genes are related to carbon metabolism, 54 genes are related to other metabolisms and 36 are the genes whose functions are unknown or unclassified (Table 1). As shown in Table 1, there is one Fnr-binding site in most of the 143 putative target genes, such as fnrl, nark, narG, and res $D$. There are two Fnr-binding sites in the promoter regions of the 16 genes, including fnr3, fur3, adhC, adhE, adhP, cah, hmp, cydA, ndh, nemA, yugK, lacI, padR, accB, yphA and yhcN. There are three Fnr-binding sites in the promoter regions of nox and $p f l B$.

The Fnr-binding motif was shown in Fig. 3A. To determine the accuracy of the bioinformatic analysis, the 13 promoter regions, including 11 putative targets with Fnr-binding sites and 2 target genes without predicted Fnr-binding sites were chosen to do electrophoretic mobility shift assays (EMAS). Fnr1 protein tagged with 6 Histidine at N-terminus (designated as NHis ${ }_{6}$-Fnr1) and Fnr3 protein tagged with 6 Histidine at C-terminus (designated as Fnr3-CHis 6 ) were expressed and purified in E. coli under aerobic condition and then the two recombinant Fur1 and Fnr3 were used in EMSA. EMSA under aerobic condition showed that both Fnr1 and Fnr3 proteins could bind to the promoter regions with the predicted Fnr-binding sites of the 8 operons: qox $A B C D$ (encoding cytochrome aa3 quinol oxidase), narGHJI (encoding nitrate reductase), $n d h$ (encoding NADH dehydrogenase), hemN3 (encoding oxygen-independent coproporphyrinogen-III oxidase), hydEG (encoding $[\mathrm{FeFe}]$ hydrogenase), $n r d D G$ (encoding anaerobic ribonucleoside triphosphate 
reductase), pflBA (encoding formate acetyltransferase), resDE (encoding two-component regulatory proteins) (Fig. 3B). However, the promoter regions of $c y d A B C D$ operon (encoding cytochrome $b d$ ubiquinol oxidase) and $n a r K$ (encoding nitrate/nitrite transporter) were only bound by Fnr3. Moreover, EMSA showed that neither Fnr1 nor Fnr3 could bind to the promoter regions of $n i f$ and $f e o A B$ (encoding the ferrous-iron transporter FeoAB), consistent with the facts that there was no Fnr-binding site in the promoter regions of these genes. However, EMSA showed that no binding of Fnr1 and Fnr3 to the promoter region of $g \ln R A$ with Fnr-binding site.

RNA-Seq transcriptome analysis of wild-type and $\Delta f$ fnr 13 strains. To assess the effects of Fnr1 and Fnr3 proteins on global gene expression in anaerobic condition, the genome-wide transcription analysis of $P$. polymyxa WLY78 and $\Delta f n r 13$ mutant cultured under $\mathrm{N}_{2}$-fixing condition (without $\mathrm{O}_{2}$ and $\mathrm{NH}_{4}{ }^{+}$) was performed. Transcripts showed statistically significant differences with q-value (p-adjusted) $\leq 0.05$ and a $\left|\log _{2} \mathrm{FC}\right| \geq 1$ were accepted as candidate differential expression genes (DEGs). Of the 5661 genes contained in the genome of $P$. polymyxa WLY78, 301 genes, including 202 genes and operon, were differentially expressed in $\Delta f n r 13$ compared to wild type (Table S2). Of the 301 genes, 116 were markedly up-regulated, indicating that they are directly or indirectly repressed by Fnr, and 185 were significantly decreased, suggesting that they were directly or indirectly activated by Fnr.

Influence of $\boldsymbol{f n r}$ genes on transcription of the nif and $g \ln R \boldsymbol{A}$ genes. The 9 genes (nifBHDKENXhesAnifV) are organized as a nif operon in P. polymyxa WLY78. In this study, we find that the expression levels of the 9 genes within the nif operon in $\Delta f n r 13$ were significantly down-regulated by 6.51-7.47 $\log _{2} \mathrm{FC}$ (Fig. 4A). The data are consistent with the decreased nitrogenase activity and nifH transcription of $\Delta f n r l 3$ mutant. However, there was no predicted Fnr-binding site in the promoter region of the nif operon and EMSA also showed that Fnr1 or Fnr3 did not bind to the promoter region of the nif gene (Fig. 3B). These results indicated that Fnr1 and Fnr3 indirectly activated the expression of nif gene operon under anaerobic conditions. Expression of $g \ln \operatorname{Rg} \ln A$ operon that plays regulatory role in nif transcription was up-regulated 1.71-1.74 $\log _{2} \mathrm{FC}$.

Influence of the $f n r 1$ and $f n r 3$ genes on transcription of the Fe transporter genes. $\mathrm{Fe}$ is an essential element for nitrogenase. $\mathrm{Fe}$ is the soluble $\mathrm{Fe}^{2+}$ form (ferrous iron) under anaerobic condition or at acidic $\mathrm{pH}$ and the major route for bacterial ferrous iron uptake was via Feo 
(Ferrous iron transport) system composed of FeoA, FeoB and FeoC $(24,25)$. Fe at neutral $\mathrm{pH}$ is bacteria excrete ferric chelators, called siderophores, to take up $\mathrm{Fe}^{3+}$. Usually, bacteria take up ferric complexes, including ferric hydroxamate (FhuCDBA), ferric citrate (YfmCDEF), ferric-haem, ferric-bacillibactin uptake system (FeuABC) (27).

Our study showed that $36 \mathrm{Fe}$ transporter genes in $\Delta f n r 13$ mutant were down-regulated 1.59-7.65 $\log _{2} \mathrm{FC}$ (Fig. 4B, Table S2). Of the $36 \mathrm{Fe}$ transporter genes, only feoAfeoB operon is involved in $\mathrm{Fe}^{2+}$ uptake and the other 34 genes belong to $\mathrm{Fe}^{3+}$ transport systems. The highest differentially expressed genes $f e o A$ and $f e o B$ were down-regulated 7.18-7.65 $\log _{2} \mathrm{FC}$. The $13 f h u$ genes belonging to ferric hydroxamate system were down-regulated from 5.32 to $1.59 \log _{2} \mathrm{FC}$. Especially, transcriptions of $y f m C D E$ involved in ferric citrate transport system and isdHCBAE involved in ferric-haem transport system were also down-regulated in $\Delta f n r 13$ mutant. The data are consistent with our recent reports that all of the $\mathrm{Fe}$ transporter genes were up-regulated in $\mathrm{N}_{2}$-fixing condition (without $\mathrm{O}_{2}$ and $\mathrm{NH}_{4}^{+}$) (28). As described above, there are no Fnr-binding sites in the promoter regions of the $36 \mathrm{Fe}$ transporter genes and EMSA also showed that Fnr1 or Fnr3 did not bind to the promoter region of the $f e o A B$ operon. Thus we deduce that Fnr1 and Fnr3 indirectly activated the expression of Fe transporter genes under anaerobic condition.

Influence of $f n r$ genes on transcription of respiration and energy metabolism genes. Based on the genome sequence, the respiratory chain of P. polymyxa WLY78 was shown in Fig. 5A. It is composed of several dehydrogenases that transfer electrons to an intramembrane pool of menaquinone and some terminal oxidases responsible for reoxidation of menaquinol. The terminal oxidases include at least two types: one consisting of a cytochrome $b d$-type quinol oxidase and the second one consisting of cytochrome aa3 oxidase.

The dehydrogenases that play an important role in respiration in Gram-positive Corynebacterium glutamicum include a non-proton-pumping NADH dehydrogenase encoded by the ndh gene, malate:quinone oxidoreductase encoded by the mqo gene, and succinate dehydrogenase encoded by the $s d h C A B$ genes (29). Here, we found that there were 13 genes encoding dehydrogenase were differentially expressed (Table S2). Of these genes, $n d h$ and 
NADH-dependent butanol dehydrogenase), hcaD (NAD(FAD)-dependent dehydrogenases), ldh

(L-lactate dehydrogenase), were up-regulated 1.76-8.44 $\log _{2} \mathrm{FC}$, while $\operatorname{glpD}$, alkH (aldehyde dehydrogenase), adhE, $f d h D$ (formate dehydrogenase) and adhP genes were obviously down-regulated 2.48-5.87 $\log _{2} \mathrm{FC}$ (Table S2). EMSA showed that NHis 6 -Fnr1 and Fnr3-CHis 6 could bind to the promoter regions of $n d h$ with the predicted Fnr-binding site (Fig. 3B). The qoxABCD encoding (cytochrome aa3-type oxidase) and cydABCD (encoding cytochrome bd-type oxidase) were up-regulated 1.8 to $4.5 \log _{2} \mathrm{FC}$.

Many bacteria are able to grow anaerobically using alternative electron acceptors, including nitrate or fumarate (30). We found that anaerobic electron acceptor genes narGHJI (nitrate reductase, Nar), nasABCD (nitrite reductase, Nas) and narK (nitrate/nitrite transporter, NarK) were down-regulated from 0.6 to 4.6 fold in $\Delta$ fnrl3 mutant. As described above, the Fnr-binding sites in the upstream region of narGHJI and narK were predicted and confirmed by EMSA. Thus, the results suggest that Fnr1 and Fnr3 directly activate expression of narGHJI and narK in anaerobiosis and indirectly activate expression of nas $A B C D$. The results are consistent with previous studies that the expression of narGHJI was intensely induced by anaerobic condition and the induction was dependent on Fnr in B. subtilis (31). In addition, the atpAFGH genes encoding ATP synthase were also down-regulated, but no Fnr-binding site was predicted in upstream regions of these genes, suggesting that Fnr1 and Fnr3 might indirectly activate expression of atpAFGH genes under anaerobic condition. These results indicated that Fnr1 and Fnr3 inhibited expression of genes involved in aerobic respiration process and activate express of genes involved in anaerobic energy metabolism. ResD-ResE (two-component regulatory proteins) and FNR were previously shown to be indispensable for nitrate respiration in $B$. subtilis $(32,33)$. Here we show that expression of resDE in $\Delta$ fnrl3 mutant were down-regulated 1.4-1.6 $\log _{2} \mathrm{FC}$, in agreement with our previous report that resD and resE were obviously up-regulated in P. polymyxaWLY78 under $\mathrm{N}_{2}$-fixation condition (without $\mathrm{NH}_{4}{ }^{+}$and $\mathrm{O}_{2}$ ) (28). EMSA Fnr1 and Fnr3 could bind to the promoter of resDE operon with a Fnr-binding site, consistent with the report that B. cereus Fnr regulated expression of resDE (38). The data indicated that Fnr1 and Fnr3 inhibited expression of the genes involved in aerobic respiratory chain and activated expression of genes responsible for anaerobic electron acceptor genes 
fixation is carried out by the enzyme nitrogenase, which transfers electrons originating from low potential electron carriers, such as flavodoxin or ferredoxin molecules, to molecular $\mathrm{N}_{2}$ (34). A flavodoxin (encoded by nifF) mediates electron transfer from a pyruvate: flavodoxin oxidoreductase (encoded by nifJ) to the Fe protein of nitrogenase in K. oxytoca (34).

At present, we do not know how many genes and which gene are involved in electron transfer for nitrogenase in P. polymyxa WLY78. The differentially expressed genes that may be the potential electron transfer for nitrogenase in P. polymyxa WLY78 were shown in Table S2 and Fig. 6A. Homology analysis showed that fldA (encoding flavodoxin) of $P$. polymyxa WLY78 showed $30 \%$ identity with $K$. oxytoca nifF. Expression of $f l d A$ in $\Delta f n r 13$ was down-regulated by 2.74 $\log _{2}$ FC. As shown in Fig. 6A, two transcripts $h y d E G$ located in plus strand and COG0196 $f d h F$ hycB hydAN aspA hydFG located in minus strand were significantly down-regulated 7.13-12.55 $\log _{2} \mathrm{FC}$. hydA encodes $\mathrm{Fe}-\mathrm{Fe}$ hydrogenase whose synthesis relies on maturation factors HydF (GTPase), HydE and HydG (35), while hydB encodes ferredoxin and $f d h F$ encodes formate dehydrogenase. Each of the promoter regions of the two operons $h y d E G$ and COG0196 $f d h F h y c B$ hydAN aspA hydFG contain a predicted Fnr binding site, and EMSA showed that NHis ${ }_{6}$-Fnr1 and Fnr3-CHis ${ }_{6}$ could bind to the promoter of hydEG (Fig. 3B). It has been reported in Clostridium, electrons produced by the oxidation of pyruvate are transferred to the acceptor ferredoxin, and then the ferredoxin can act as electron donors to reduce $\mathrm{Fe}-\mathrm{Fe}$ hydrogenase HydA to produce hydrogen (36). In addition, the expression of hemN1, hemN3 (hemN encoded oxygen-independent coproporphyrinogen-III oxidase) and COG1249 (encoded FAD-dependent oxidoreductase) were also down-regulated 2.40-9.61 $\log _{2}$ FC. EMSA showed that Fnr1 and Fnr3 could bind to the promoter of hemN3 with a predicted Fnr binding site. Furthermore, we found that hmp (flavohemoprotein), $w r b A$ (multimeric flavodoxin), $y w n B$ (NADH-flavin reductase), $r i b E$ (riboflavin synthase) and groSgroL (chaperonin) were up-regulated 1.26-6.88 $\log _{2}$ FC. EMSA showed that Fnr1 and Fnr3 could bind to the promoter of hmp with two Fnr binding sites. We deduce that some of the differentially expressed genes, including fldA (flavodoxin and hydB (ferredoxin) may be involved in be the potential electron transfer for nitrogenase in $P$. polymyxa WLY78. However, some genes involved in electron transfer for nitrogenase, such as $n f r A$ (encoding NAD(P)H Flavin oxidoreductase), were not differentially expressed. 
involved in carbon metabolism (such as glycolysis, the Krebs (TCA) cycle and fermentation) were shown in Fig. 6C and Table S2. The down-regulated (1.33-3.28 $\log _{2} \mathrm{FC}$ ) genes in $\Delta$ fnrl3 were involved in glycolysis. These genes included ptsG (encoding glucose-specific component in PTS system), $\quad p f k A \quad$ (encoding ATP-dependent 6-phosphofructokinase), fbaA (encoding fructose-bisphosphate aldolase), pgm (encoding $\beta$-phosphoglucomutase) and pykA (encoding pyruvate kinase). However, Fnr-binding sites were not found in the upstream regions of these genes, suggesting that Fur indirectly affected expression of the genes involved in glycolysis.

Many genes participated in formate and ethanol metabolism were significantly down-regulated in $\Delta f n r l 3$ mutant, such as $p f l B A$ (encoding formate acetyl transferase), $f d h D$ (encoding formate dehydrogenase), $a d h E$ (encoding aldehyde-alcohol dehydrogenase) and $a l k H$ (encoding aldehyde dehydrogenase). Multiple Fnr-binding sites in the upstream regions of $p f l B A$ and $a d h E$ were predicted and EMAS also showed the binding of Fnr1 and Fnr3 to the promoter of pflBA (Table1 and Fig. 3B). This implied that Fnr1 and Fnr3 might have a direct regulation in expression of these genes under anaerobic condition. In contrast, $l d h$ encoding L-lactate dehydrogenase was significantly up-regulated by 6.1 fold, but there was no predicted Fnr-binding site in the promoter region of this gene.

Many genes in the Krebs cycle were significantly up-regulated from 1.21 fold to 4.21 fold, and they included citZ (encoding citrate synthase), citB (encoding aconitate hydratase), icd (encoding isocitrate dehydrogenase), $o d h A B$ (encoding 2-oxoglutarate dehydrogenase), sucCD (encoding succinyl-CoA synthase) and $\operatorname{sdh} A B C$ (encoding succinate dehydrogenase). However, there were no predicted Fnr-binding sites in the upstream region of these genes. These data suggested that Fnr1 and Fnr3 indirectly activated expression of genes involved in glycolysis and indirectly inhibited expression of genes involved in the Krebs cycle in P. polymyxa WLY78.

\section{DISCUSSION}

Fnr is a global transcriptional regulator that controls a lot of genes expression in response to the transition from aerobic to anaerobic conditions in many bacteria. Although Fnr is well known in $E$. coli and B. subtilis, the function of Fnr in Paenibacillus, especially in $\mathrm{N}_{2}$-fixing Paenibacillus, is not known. P. polymyxa WLY78 that fixes nitrogen in anaerobic or microaerobic conditions has four $f n r$ genes. Here, the functions of the fnr genes of $P$. polymyxa WLY78 in nitrogen fixation and 
other metabolisms were investigated.

We found that like $P$. polymyxa WLY78, P. polymyxa M1, P. polymyxa E681 and P. polymyxa SC2 have four fnr genes and each of the four fnr genes exhibited more than $90 \%$ identity with its corresponding $f n r$, suggesting that these bacterial strains have a common fnr gene ancestor. Whereas, some Paenibacillus species and strains, such as $P$. polymyxa EBL06, P. polymyxa Sb3-1 and P. jamilae NS115 have only a fnr gene whose predicted product Fnr shows higher (34.78\%) identity with Fnr7 than with Fnr1, Fnr3 and Fnr5, suggesting that Fnr7 is conserved in Paenibacillus. However, Fnr1 and Fnr3 of P. polymyxa WLY78 have high similarity with both $B$. subtilis Fnr and B. cereus Fnr in sequence and structure, suggesting that Fnr1 and Fnr3 of $P$. polymyxa WLY78, B. subtilis Fnr and B. cereus Fnr have a common fnr gene ancestor.

Deletion of fnrl and fnr3 genes of P. polymyxa WLY78 resulted to about $50 \%$ decrease of both growth rate and nitrogenase activity under anaerobic condition. Deletion of fnr5 and $f n r 7$ genes led to a slight decrease of both growth rate and nitrogenase activity under anaerobic condition. The data suggest that the $f n r l$ and $f n r 3$ genes play important roles in growth and nitrogen fixation under anaerobic conditions. However, the growth rates and nitrogenase activities of the multiple deletion mutants, such as $\Delta f n r 17, \Delta f n r 57$ and $\Delta f n r 357$, were higher than single deletion mutants. The data implied that there might be some interactions among the four Fur proteins. Recently, specific interaction between Fnr1 and Fnr3 of $H$. seropedicae has been determined by using two-hybrid assays (37). Fnr1 and Fnr3 of H. seropedicae directly regulate discrete groups of promoters (Groups I and II, respectively), while Fnr3-Fnr1 heterodimers regulate a third group (Group III) promoters (37). Whether heterodimer is formed between Fnr1 or Fnr3 with Fnr5 or Fnr7 of $P$. polymyxa WLY78 needs to be studied in the near future.

In this study, Fnr1 with $\mathrm{His}_{6}$-tags at its $\mathrm{N}$-terminus and Fnr3 with $\mathrm{His}_{6}$-tags at its C-terminus were expressed and purified in E. coli under aerobic condition. Both of the purified recombinant protein solutions did not exhibit the characteristic brown color, suggesting that $\mathrm{Fe}_{4}-\mathrm{S}_{4}$ cluster was oxidized by $\mathrm{O}_{2}$. However, EMSA showed that His-tagged Fnr1 and His-tagged Fnr3 of $P$. polymyxa WLY78 could bind to the promoter regions with the Fnr-binding site (5'-TGTGA-N6-TCACA-3'). Binding to the specific promoters suggest that the aerobically purified Fnr1 and Fnr3 proteins of $P$. polymyxa WLY78 were active forms. Similar report was found that both $B$. cereus Fnr tagged with His at its C-terminus $\left(\mathrm{Fnr}_{\mathrm{His}}\right)$ and Fnr tagged with Strep 
at its N-terminus (StrepFnr) were active when expressed and purified in E. coli under oxic conditions. In vitro, the aerobically purified B. cereus Fnr as a monomer bound to the promoter regions of $f n r$ itself, $r e s D E$, $p l c R$ and the structural enterotoxin genes $h b l$ and $n h e(38)$. Unlike $B$. cereus Fnr, B. subtilis Fnr existed in an inactive state under aerobiosis, due to the $[4 \mathrm{Fe}-4 \mathrm{~S}]^{2+}$ cluster of FNR being converted by $\mathrm{O}_{2}$ to a $[2 \mathrm{Fe}-2 \mathrm{~S}]^{2+}$. The B. subtilis Fnr formed stable dimer under aerobic and anaerobic conditions independent of Fe-S cluster formation, but DNA binding of Fnr was dependent on the presence of intact $[4 \mathrm{Fe}-4 \mathrm{~S}]^{2+}$ cluster (11). As a member of CRP/FNR family transcription factors, Fnr should function as a dimer in vivo. It is known that many transcription factors as a dimer bind to its specific DNA sites and there are two pathways to form dimeric protein-DNA complexes. Dimer pathway implies that the protein can dimerize first and then associate with DNA, and monomer pathway means that two protein monomers bind DNA sequentially and form their dimerization interface while bound to DNA (39). It was proposed that $B$. cereus Fnr takes a sequential monomer-binding pathway to form a dimer. But $B$. subtilis Fnr as a homodimer binds to its specific DNA-binding site and activates transcription (11). Our results suggest that Fnr1 and Fnr3 of P. polymyxa WLY78 behaved as B. cereus Fnr did. Thus, we deduce that in vivo Fnr1 and Fnr3 proteins of $P$. polymyxa WLY78 may bind to the specific promoter region by a sequential monomer-binding pathway just as B. cereus Fnr did.

Genome-wide transcription analysis showed that 301 genes, including 202 genes and operons, were differentially expressed in $\Delta f n r 13$ compared to $P$. polymyxa WLY78 (Table S2). Similar reports were found that E. coli Fnr controlling the synthesis of up to 125 genes (40). Of the 301 genes, 116 were markedly up-regulated, indicating that they were directly or indirectly repressed by Fnr, and 185 were significantly down-regulated, suggesting that they were directly or indirectly activated by Fnr. Notably, the 9 genes (nifBHDKENXhesAnifV) within the nif operon in $P$. polymyxa WLY78 were significantly down-regulated 6.51-7.47 $\log _{2} \mathrm{FC}$. The data were consistent with the decreased nitrogenase activity of $\Delta f n r l 3$ mutant. qRT-PCR also confirmed that the nifH transcription in $\Delta f n r l 3$ mutant was obviously reduced. However, there was no predicted Fnr-binding site in the promoter region of the nif operon and EMSA also showed that Fnr1 or Fnr3 did not bind to the promoter region of the nif genes. These results indicated that Fnr1 and Fnr3 
and nitrogen-limited condition. However, $g \ln R g \ln A$ operon was up-regulated in $\Delta f n r l 3$ mutant, suggesting that $g \ln R$ expression was not in coordination with nif expression. Also, EMSA showed that there was no binding of Fnr1 or Fnr3 to the promoter region of $g \ln R$ with an Fnr-binding site. We do not know whether Fnr5 or Fnr7 could bind to the promoter region of $g \ln R$. In contrast to our results, the combined deletions in both the fnrl and fnr3 genes in $H$. seropedicae led to higher expression of nifA, nifB and nifH, which was probably as a consequence of their influence on respiratory activity in relation to oxygen availability (16). It was shown that Fnr was required for relief of NifL inhibition in K. oxytoca under anaerobic conditions (14).

$\mathrm{Fe}$ is an essential element for nitrogenase. Our study showed that $36 \mathrm{Fe}$ transporter genes in $\Delta$ fnrl3 mutant were significantly down-regulated compared to wild type. But there were no Fnr-binding sites in the promoter regions of these genes, suggesting that Fnr1 and Fnr3 indirectly induced expression of genes involved in uptake of Fe. Of the $36 \mathrm{Fe}$ transporter genes, only feoAfeoB were involved in $\mathrm{Fe}^{2+}$ uptake and the other 34 genes belonged to $\mathrm{Fe}^{3+}$ transport systems. We deduce that the two forms of $\mathrm{Fe}^{2+}$ and $\mathrm{Fe}^{3+}$ coexisted in the culture of P. polymyxa WLY78 grown in anaerobic condition at neutral $\mathrm{pH}$ and then both types of $\mathrm{Fe}^{2+}$ and $\mathrm{Fe}^{3+}$ uptake systems were induced by Fnr. But we do not know how Fnr indirectly induced expression of genes involved in uptake of $\mathrm{Fe}^{2+}$ and $\mathrm{Fe}^{3+}$.

Since nitrogenase is very sensitive to oxygen, nitrogen fixation under anaerobic or microaerobic conditions. We found that the $n d h$ gene (NADH dehydrogenase) and the $s d h C A B$ genes (succinate dehydrogenase) that are the major dehydrogenase genes in the respiratory chain in $\Delta$ fnrl3 mutant were up-regulated under anaerobic condition. Other dehydrogenase genes, such as $y u t J$ (NADH dehydrogenase), yugK (Probable NADH-dependent butanol dehydrogenase), $h c a D$ (NAD(FAD)-dependent dehydrogenases) and ldh (L-lactate dehydrogenase), were up-regulated. On the contrary, anaerobic electron acceptor genes narGHJI (nitrate reductase, Nar), nas $A B C D$ (nitrite reductase, Nas) and narK (nitrate/nitrite transporter, NarK) were down-regulated in $\triangle f n r 13$ mutant. Importantly, expression of resDE whose promoter has an Fnr-binding site was down-regulated under anaerobic condition. The direct regulation of resDE by Fur was also reported in B. cereus. The data indicated that Fnr1 and Fnr3 inhibited expression of the genes involved in aerobic respiratory chain, and activated expression of anaerobic electron acceptor genes. These results also suggest that Fnr1 and Fnr3 provided $\mathrm{O}_{2}$ protection and energy for 
nitrogen fixation under anaerobic condition.

Nitrogen fixation is a process in which electrons originating from low potential electron carriers, such as flavodoxin or ferredoxin molecules were transferred to molecular $\mathrm{N}_{2}$. In $K$. oxytoca, the electron was produced by pyruvate: flavodoxin oxidoreductase (encoded by nifJ) during tricarboxylic acid cycle (TCA) and then a flavodoxin (encoded by niff) mediated electron transfer to the Fe protein of nitrogenase (34). At present, we do not know the specific electron transfer system for nitrogen fixation in $P$. polymyxa WLY78. According to our previous study, $P$. polymyxa has several genes encoding flavodoxin or ferredoxin or oxidoreductase may be involved in electron transfer to nitrogenase. Here, we showed that $f l d A$ (flavodoxin), fldB (flavodoxin), flr (flavoredoxin), $y d f E$ (flavoprotein oxygenases), porG porA (pyruvate:ferredoxin oxidoreductase), $y w c H 3$ (flavin-dependent oxidoreductases) and $y w c H 1$ (flavin-dependent oxidoreductases) were not differentially expressed. But fldA (flavodoxin), hydA (Fe-Fe hydrogenase), hemNl and hemN3 (coproporphyrinogen-III oxidase) were down-regulated, suggesting that these genes may play important role in transferring electron to nitrogenase.

Taken together, the copy numbers of the fnr gene vary among different Paenibacillus species and different $P$. polymyxa strains. $P$. polymyxa WLY78 has four fnr genes encoding a global anaerobic regulator. The Fnr7 was conserved in different Paenibacillus species and strains. Fnr1 and Fnr3 of P. polymyxa WLY78 has more similarity to each other than to Fnr5 and Fnr7. Fnr1 and Fnr3 of P. polymyxa WLY78 also has high similarity with B. subtilis Fnr and B. cereus Fnr. EMSA showed that the aerobically purified Fnr1 and Fnr3 could bind to the specific target DNA in vitro as B. cereus Fnr did. We deduce that in vivo Fnr1 and Fnr3 of $P$. polymyxa WLY78 may bind to the specific promoter region by a sequential monomer-binding pathway to form a complex of a dimeric protein and DNA. Deletion of $f n r l$ and $f n r 3$ led to a significant decrease of nitrogenase activity under anaerobic condition. Transcriptional analysis showed that Fnr1 and Fnr3 indirectly activate expression of the nif gene and Fe transported genes under anaerobic condition. Fnr1 and Fnr3 inhibit expression of the genes involved in aerobic respiratory chain and activate expression of genes responsible for anaerobic electron acceptor genes, which might provide $\mathrm{O}_{2}$ protection and energy for nitrogenase. In addition to Fnr1 and Fnr3, the function of Fnr5 and Fnr7 need to be studied in the future. This study not only reveals the roles of fnr genes in nitrogen fixation and electron transport, but also will provide a clue to clarifying the regulatory 
mechanisms of Fnr in nitrogen fixation in response to $\mathrm{O}_{2}$.

\section{MATERIALS AND METHODS}

Strains and media. $P$. polymyxa WLY78 used here was isolated from rhizosphere of bamboo by our laboratory (41). P. polymyxa and $\Delta f n r$ mutants were routinely grown at $30^{\circ} \mathrm{C}$ in $\mathrm{LB}$ or LD medium (per liter contains: $5 \mathrm{~g} \mathrm{NaCl}, 5 \mathrm{~g}$ yeast and $10 \mathrm{~g}$ tryptone) with shaking. When appropriate, antibiotics were added in the following concentrations: $12.5 \mathrm{mg} / \mathrm{ml}$ tetracycline, $5 \mathrm{mg} / \mathrm{ml}$ erythromycin and $100 \mathrm{mg} / \mathrm{ml}$ ampicillin for maintenance of plasmids.

Nitrogen-deficient media were used for assay of nitrogenase activity. Nitrogen-deficient medium contained (per liter) $10.4 \mathrm{~g} \mathrm{Na}_{2} \mathrm{HPO}_{4}, 3.4 \mathrm{~g} \mathrm{KH}_{2} \mathrm{PO}_{4}, 26 \mathrm{mg} \mathrm{CaCl}{ }_{2}^{\bullet} 2 \mathrm{H}_{2} \mathrm{O}, 30 \mathrm{mg} \mathrm{MgSO}$, $0.3 \mathrm{mg} \mathrm{MnSO}_{4}, 36 \mathrm{mg}$ Ferric citrate, $7.6 \mathrm{mg} \mathrm{Na} 2 \mathrm{MoO}_{4} \cdot 2 \mathrm{H}_{2} \mathrm{O}, 10 \mu \mathrm{g}$ p-aminobenzoic acid, $5 \mu \mathrm{g}$ biotin, $4 \mathrm{~g}$ glucose as carbon source and $2 \mathrm{mM}$ glutamate as nitrogen source (41).

Nitrogenase activity assays. For nitrogenase activity assays, $P$. polymyxa WLY78 and $\Delta f n r$ mutants were grown in $50 \mathrm{ml}$ LD media (supplemented with antibiotics when necessary) in 250 $\mathrm{ml}$ test tubes shaken at $250 \mathrm{rpm}$ for $16 \mathrm{~h}$ at $30{ }^{\circ} \mathrm{C}$. The cultures were collected by centrifugation, washed three times with sterilized water and then resuspended in nitrogen-deficient medium containing $2 \mathrm{mM}$ glutamate to a final $\mathrm{OD}_{600}$ of $0.3-0.5$. Then, $3-5 \mathrm{ml}$ of suspension was transferred to a $26 \mathrm{ml}$ test tube which was sealed with rubber stopper. The headspace in the tube was then vacuumed and filled with argon gas (42). After $\mathrm{C}_{2} \mathrm{H}_{2}$ (10\% of the headspace volume) was injected into the test tubes, the cultures were incubated at $30^{\circ} \mathrm{C}$ and with shaking at $250 \mathrm{rpm}$. After incubating for 4-8 h, $100 \mu \mathrm{l}$ of gas was withdrawn through the rubber stopper with a gas tight syringe and manually injected into the gas chromatograph (HP6890) to quantify ethylene production. All treatments were in three replicates and all the experiments were repeated three or more times.

$\boldsymbol{\beta}$-galactosidase assays. To confirm whether deletion of fnr genes affect nif gene transcription, $P$. polymyxa WLY78 and 12 fnr mutants were transformed with a recombinant plasmid carrying the nif promoter-lacZ fusion (Pnif-lacZ fusion) (19). $\beta$-galactosidase activity was assayed according to the method described by Wang et al (19).

Identification and sequence alignment of P. polymyxa Fnr proteins. The sequences of Fnr1, Fnr3, Fnr5 and Fnr7 from P. polymyxa WLY78 were aligned with that of the Fnr of Bacillus subtilis subsp. subtilis str. 168 (Ref seq: NP_391612.1) and Bacillus cereus F4430-73 (Ref seq: 
507 KMP55664.1) using Clustal W software. The conserved domains in the Fnr proteins were 508 investigated by sequence searching to the Pfam database (http://pfam.sanger.ac.uk/). The 509 secondary structure elements in the Fnr proteins were defined by ESPript 3.0 algorithm (43).

510 Phylogenetic analysis. In the non redundant NCBI database, amino acid sequences were obtained 511 by performing a BLASTP search. Multiple gene alignments were carried out with molecular 512 evolutionary genetics analysis (MEGA) (44). The neighbor-joining trees were constructed and 5131,000 bootstraps were done by using the MEGA 7.0.14 software.

514 Construction of $\Delta f n r$ mutants. Here, $12 \Delta f n r$ mutants, including single mutants $\Delta f n r l, \Delta f n r 3$, $\Delta f n r 5$ and $\Delta f n r 7$, double mutants $\Delta f n r 13, \Delta f n r 17, \Delta f n r 35, \Delta f n r 37$ and $\Delta f n r 57$, triple mutants $\Delta f n r 137$ and $\Delta f n r 357$, and quadruple mutants $\Delta f n r 1357$, were constructed. The unmarked, single and multiple $f n r$ deletion mutants were constructed via homologous recombination using the suicide plasmid pRN5101 as described previously (19). The upstream and downstream fragments flanking the coding region of fnrl, fnr3 fnr5 and $f n r 7$ were PCR amplified from the genomic DNA of $P$. polymyxa WLY78, respectively. The primers used for deletion mutagenesis were listed in Table S3. The upstream and downstream fragments of four fnr genes were then fused with BamH I /HindIII digested vector pRN5101 in Gibson assembly master mix (New England Biolabs), generating the four recombinant plasmids. Then, each of these recombinant plasmids was transformed into $P$. polymyxa WLY78 as described by (19), and the single crossover transformants were selected for erythromycin resistance $\left(\mathrm{Em}^{\mathrm{r}}\right)$. Subsequently, marker-free deletion mutants (the double-crossover transformants) were selected from the initial $\mathrm{Em}^{\mathrm{r}}$ transformants $^{-1}$ after several rounds of nonselective growth at $39^{\circ} \mathrm{C}$. The marker-free deletion mutants were confirmed by PCR amplification and DNA sequencing analysis. The multiple fur deletion mutants were constructed via the same method in the single fnr deletion mutant background. were PCR amplified from the genomic DNA of P. polymyxa WLY78, respectively. These PCR products were cloned into pET-28b(+) (Novagen, USA) to construct tagged Fnr proteins with His-tag at the N-terminus of Fnr1 and C-terminus of Fnr3 respectively, and then transformed into 534 E. coli BL21 (DE3). The recombinant E. coli strains were cultivated at $37^{\circ} \mathrm{C}$ in $\mathrm{LB}$ broth supplemented with $50 \mu \mathrm{g} / \mathrm{ml}$ kanamycin until midlog phase, when $0.2 \mathrm{mM}$ IPTG was added and incubation continued at $16^{\circ} \mathrm{C}$ for 8 hours. Cells were collected and disrupted in the lysis buffer (50 
$\mathrm{mM} \mathrm{NaH}{ }_{2} \mathrm{PO}_{4}, 300 \mathrm{mM} \mathrm{NaCl}, 10 \mathrm{mM}$ Imidazole) by sonication on ice. Recombinant proteins $\mathrm{NHis}_{6}$-Fnr1 and Fnr3-CHis 6 in the supernatant were purified on $\mathrm{Ni}_{2}$-NTA resin (Qiagen, Germany) according to the manufacturer's protocol. Fractions eluted with $250 \mathrm{mM}$ imidazole were dialyzed into binding buffer (20 mM HEPES pH 7.6, 1mM EDTA, $10 \mathrm{mM}\left(\mathrm{NH}_{4}\right)_{2} \mathrm{SO}_{4}, 1 \mathrm{mM}$ DTT, $0.2 \%$ Tween 20, $30 \mathrm{mM} \mathrm{KCl}$ ) for electrophoretic mobility shift assays (EMSA). Primers used here were listed in Table S3.

Electrophoretic mobility shift assays (EMSAs). EMSAs were performed as described previously using a DIG Gel Shift Kit (2nd Generation, Roche, USA) (19). The promoter fragments of predicted target genes or operons were PCR amplified from the genomic DNA of $P$. polymyxa WLY78. The primers used here and DNA fragment sizes were listed in Table S2. The DNA fragments were labeled at the 3' end with digoxigenin (DIG) using terminal transferase, and used as probes in EMSAs. Each binding reaction $(20 \mu \mathrm{l})$ consisted of $0.3 \mathrm{nM}$ labelled probe, $1 \mu \mathrm{g}$ poly $[\mathrm{d}(\mathrm{A}-\mathrm{T})]$ and various concentrations $(0,0.05,0.2,2,6 \mu \mathrm{M})$ of purified His-tagged Fnr (apo-Fnr) in the binding buffer. Reaction mixtures were incubated for $30 \mathrm{~min}$ at $25^{\circ} \mathrm{C}$, analyzed by electrophoresis using native $5 \%$ polyacrylamide gel run with $0.5 \times \mathrm{TBE}$ as running buffer at $4^{\circ} \mathrm{C}$, and electrophoretically transferred to a positively charged nylon membrane (GE healthcare, UK). Labelled DNAs were detected by chemiluminescence according to the manufacturer's instructions, and recorded on X-ray film.

Bacterial RNA extraction and transcriptomic analysis. P. polymyxa WLY78 WT and $\Delta f n r 13$ mutant were grown in nitrogen-deficient medium under anaerobic condition in $250 \mathrm{ml}$ test tubes shaken at $250 \mathrm{rpm}$ for $8 \mathrm{~h}$ at $30^{\circ} \mathrm{C}$. The cultures were quickly collected by centrifugation at $4^{\circ} \mathrm{C}$ under anaerobic condition and stored in liquid nitrogen for further use. This experiment was repeated three times.

For bacterial RNA extraction, bacterial cultures at each experimental time point were harvested and rapidly frozen in liquid nitrogen. Total RNAs were extracted with RNAiso Plus (Takara, Japan) according to the manufacturer's protocol. Removal of genomic DNA and synthesis of cDNA were performed using PrimeScript RT reagent Kit with gDNA Eraser (Takara, Japan). The concentration of purified RNA was quantified on a Nanodrop ND-1000 spectophotometer (NanoDrop Technologies, Thermo FisherScientific, USA). 

protocol. Differential expression analysis of two groups (two biological replicates per condition) was performed using the DESeq R package (1.18.0) (45). DESeq provides statistical routines for determining differential expression in digital gene expression data using a model based on the negative binomial distribution. The resulting P-values were adjusted using the Benjamini and Hochberg's approach for controlling the false discovery rate. The differences of transcript level with an adjusted P-value $<0.05$ determined by DESeq were considered to be significant and the genes were assigned as differentially expressed genes (DEGs). The DEGs were annotated using KEGG (Kyoto Encyclopedia of Genes and Genomes) database (http://www.genome.jp/kegg/). Gene Ontology (GO) enrichment analysis of differentially expressed genes was implemented by the GOseq R package, in which gene length bias was corrected. All of the raw reads are archived at the NCBI Sequence Read Archive (SRA) database (accession number: PRJNA596607). Transcriptional analysis was performed in triplicate and the reproducibility of the biological repeats was high (a mean $\mathrm{R}^{2}=0.929$ ).

qRT-PCR analysis. Transcription levels of genes among $P$. polymyxa WLY78 and fur deletion mutants were compared by quantitative real-time RT-PCR (qRT-PCR) analysis. Primers used for qRT-PCR are listed in Table S3. qRT-PCR was performed on Applied Biosystems 7500 Real-Time System (Life Technologies, USA) and detected by the SYBRGreen detection system with the following program: $95^{\circ} \mathrm{C}$ for $15 \mathrm{~min}, 1$ cycle; $95^{\circ} \mathrm{C}$ for 10 sand $65^{\circ} \mathrm{C}$ for $30 \mathrm{~s}, 40$ cycles. The relative expression level was calculated using $\Delta \Delta \mathrm{Ct}$ method and 16S rRNA was set as internal control. Triplicate assays using RNAs extracted in three independent experiments were performed for each target gene.

\section{Acknowledgments}

590 This work was supported by the China Natural National Science Foundation (Grant No. 31770083)

591 and the Tianjin Innovative Experimental Project for Young researchers (Grant No. 2020001).

\section{References}

593 1. Tezcan, F. A., J. T. Kaiser, D. Mustafi, M. Y. Walton, J. B. Howard, and D. C. Rees. 2005. 594 Nitrogenase complexes: multiple docking sites for a nucleotide switch protein. Science 
2. Goldberg, I., V. Nadler, and A. Hochman. 1987. Mechanism of nitrogenase switch-off by oxygen. J Bacteriol 169:874-879.

3. Robson, R. L., and J. R. Postgate. 1980. Oxygen and hydrogen in biological nitrogen fixation. Annu Rev Microbiol 34:183-207. https://doi.org/10.1146/annurev.mi.34.100180.001151.

4. Oelze, J. 2000. Respiratory protection of nitrogenase in Azotobacter species: is a widely held hypothesis unequivocally supported by experimental evidence? FEMS Microbiol Rev 24:321-333. https://doi.org/10.1111/j.1574-6976.2000.tb00545.x.

5. Murry, M. A., A. J. Horne, and J. R. Benemann. 1984. Physiological Studies of Oxygen Protection Mechanisms in the Heterocysts of Anabaena cylindrica. Appl Environ Microbiol 47:449-454.

6. Myers, K. S., H. Yan, I. M. Ong, D. Chung, K. Liang, F. Tran, S. Keles, R. Landick, and P. J. Kiley. 2013. Genome-scale analysis of Escherichia coli FNR reveals complex features of

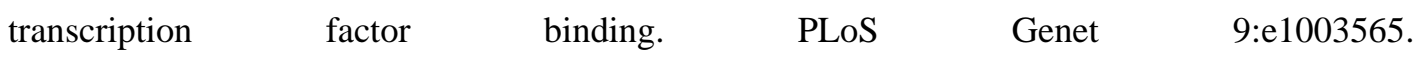
https://doi.org/10.1371/journal.pgen.1003565.

7. Reents, H., R. Munch, T. Dammeyer, D. Jahn, and E. Hartig. 2006. The Fnr regulon of Bacillus subtilis. J Bacteriol 188:1103-1112. https://doi.org/10.1128/JB.188.3.1103-1112.2006.

8. Zhou, D., and R. Yang. 2006. Global analysis of gene transcription regulation in prokaryotes. Cell Mol Life Sci 63:2260-2290. https://doi.org/10.1007/s00018-006-6184-6.

9. Martinez-Antonio, A., and J. Collado-Vides. 2003. Identifying global regulators in transcriptional regulatory networks in bacteria. Curr Opin Microbiol 6:482-489. https://doi.org/10.1016/j.mib.2003.09.002.

10. Kiley, P. J., and H. Beinert. 1998. Oxygen sensing by the global regulator, FNR: the role of the iron-sulfur cluster. FEMS Microbiol Rev 22:341-52. https://doi.org/10.1111/j.1574-6976.1998.tb00375.x.

11. Reents, H., I. Gruner, U. Harmening, L. H. Bottger, G. Layer, P. Heathcote, A. X. Trautwein, D. Jahn, and E. Hartig. 2006. Bacillus subtilis Fnr senses oxygen via a [4Fe-4S] cluster coordinated by three cysteine residues without change in the oligomeric state. Mol Microbiol 60:1432-1445. https://doi.org/10.1111/j.1365-2958.2006.05198.x.

12. Mesa, S., F. Hauser, M. Friberg, E. Malaguti, H. M. Fischer, and H. Hennecke. 2008. Comprehensive assessment of the regulons controlled by the FixLJ-FixK2-FixK1 cascade in 

response and late symbiotic functions of Sinorhizobium meliloti. J Bacteriol 188:4890-4902. https://doi.org/10.1128/JB.00251-06.

14. Grabbe, R., K. Klopprogge, and R. A. Schmitz. 2001. Fnr Is required for NifL-dependent oxygen control of nif gene expression in Klebsiella pneumoniae. J Bacteriol 183:1385-1393. https://doi.org/10.1128/JB.183.4.1385-1393.2001.

15. Gutierrez, D., Y. Hernando, J. M. Palacios, J. Imperial, and T. Ruiz-Argueso. 1997. FnrN controls symbiotic nitrogen fixation and hydrogenase activities in Rhizobium leguminosarum biovar viciae UPM791. J Bacteriol 179:5264-5270. https://doi.org/10.1128/jb.179.17.5264-5270.1997.

16. Batista, M. B., R. Wassem, F. O. Pedrosa, E. M. de Souza, R. Dixon, and R. A. Monteiro. 2015. Enhanced oxygen consumption in Herbaspirillum seropedicae fnr mutants leads to increased NifA mediated transcriptional activation. BMC Microbiol 15:95. https://doi.org/10.1186/s12866-015-0432-6.

17. Batista, M. B., M. Z. Sfeir, H. Faoro, R. Wassem, M. B. Steffens, F. O. Pedrosa, E. M. Souza, Aspartate 141 is the fourth ligand of the oxygen-sensing $[4 \mathrm{Fe}-4 \mathrm{~S}]^{2+}$ cluster of Bacillus subtilis transcriptional regulator Fnr. Biol Chem 286:2017-2021. 
https://doi.org/10.1074/jbc.M110.191940.

21. Schollhorn, R., and R. H. Burris. 1967. Acetylene as a competitive inhibitor of N-2 fixation. Proc Natl Acad Sci U S A 58:213-216.

22. Dilworth, M. J. 1966. Acetylene reduction by nitrogen-fixing preparations from Clostridium pasteurianum. Biochim Biophys Acta 127:285-294.

23. Li, X., J. Wang, S. Li, J. Ji, W. Wang, and K. Yang. 2015. ScbR- and ScbR2-mediated signal transduction networks coordinate complex physiological responses in Streptomyces coelicolor. Sci Rep 5:14831. https://doi.org/10.1038/srep14831.

24. Cartron, M. L., S. Maddocks, P. Gillingham, C. J. Craven, and S. C. Andrews. 2006. Feo-transport of ferrous iron into bacteria. Biometals 19:143-157. https://doi.org/10.1007/s10534-006-0003-2.

25. McHugh, J. P., F. Rodriguez-Quinones, H. Abdul-Tehrani, D. A. Svistunenko, R. K. Poole, C. E. Cooper, and S. C. Andrews. 2003. Global iron-dependent gene regulation in Escherichia coli. A new mechanism for iron homeostasis. J Biol Chem 278:29478-29486. https://doi.org/10.1074/jbc.M303381200.

26. Guerinot, M. L. 1994. Microbial iron transport. Annu Rev Microbiol 48:743-772. https://doi.org/10.1146/annurev.mi.48.100194.003523.

27. Andrews, S. C., A. K. Robinson, and F. Rodriguez-Quinones. 2003. Bacterial iron homeostasis. FEMS Microbiol Rev 27:215-237. https://doi.org/10.1016/S0168-6445(03)00055-X.

28. Shi, H. W., L. Y. Wang, X. X. Li, X. M. Liu, T. Y. Hao, X. J. He, and S. F. Chen. 2016. Genome-wide transcriptome profiling of nitrogen fixation in Paenibacillus sp. WLY78. BMC Microbiol 16:25. https://doi.org/10.1186/s12866-016-0642-6.

29. Niebisch, A., and M. Bott. 2003. Purification of a cytochrome $b c_{1}-a a_{3}$ supercomplex with quinol oxidase activity from Corynebacterium glutamicum. Identification of a fourth subunity of cytochrome $a a_{3}$ oxidase and mutational analysis of diheme cytochrome $c_{1}$. J Biol Chem 278:4339-46. https://doi.org/10.1074/jbc.M210499200.

30. Glaser, P., A. Danchin, F. Kunst, P. Zuber, and M. M. Nakano. 1995. Identification and isolation of a gene required for nitrate assimilation and anaerobic growth of Bacillus subtilis. J Bacteriol 177:1112-5.

31. Nakano, M. M., and P. Zuber. 1998. Anaerobic growth of a "strict aerobe" (Bacillus subtilis). 
Annu Rev Microbiol 52:165-190. https://doi.org/10.1146/annurev.micro.52.1.165.

32. Hoffmann, T., B. Troup, A. Szabo, C. Hungerer, and D. Jahn. 1995. The anaerobic life of Bacillus subtilis: cloning of the genes encoding the respiratory nitrate reductase system. FEMS Microbiol Lett 131:219-25. https://doi.org/10.1111/j.1574-6968.1995.tb07780.x.

33. Cruz, R. H., L. Boursier, I. Moszer, F. Kunst, A. Danchin, and P. Glaser. 1995. Anaerobic transcription activation in Bacillus subtilis: identification of distinct FNR-dependent and -independent regulatory mechanisms. EMBO J 14:5984-5994.

34. Hill, S., and E. P. Kavanagh. 1980. Roles of nifF and nifJ gene products in electron transport to nitrogenase in Klebsiella pneumoniae. J Bacteriol 141:470-475.

35. Posewitz, M. C., P. W. King, S. L. Smolinski, L. Zhang, M. Seibert, and M. L. Ghirardi. 2004. Discovery of two novel radical S-adenosylmethionine proteins required for the assembly of an active $\quad[\mathrm{Fe}] \quad$ hydrogenase. $\quad \mathrm{J} \quad$ Biol $\quad$ Chem 279:25711-25720. https://doi.org/10.1074/jbc.M403206200.

36. Akhtar, M. K., and P. R. Jones. 2009. Construction of a synthetic YdbK-dependent pyruvate: $\mathrm{H}_{2}$ pathway in Escherichia coli BL21 (DE3). Metab Eng 11:139-147. https://doi.org/10.1016/j.ymben.2009.01.002.

37. Batista, M. B., G. Chandra, R. A. Monteiro, E. M. de Souza, and R. Dixon. 2018. Hierarchical interactions between Fnr orthologs allows fine-tuning of transcription in response to oxygen in Herbaspirillum seropedicae. Nucleic Acids Res 46:3953-3966. https://doi.org/10.1093/nar/gky142.

38. Esbelin, J., Y. Jouanneau, J. Armengaud, and C. Duport. 2008. ApoFnr binds as a monomer to promoters regulating the expression of enterotoxin genes of Bacillus cereus. $\mathrm{J}$ Bacteriol 190:4242-51. https://doi.org/10.1128/JB.00336-08.

39. Kohler, J. J., S. J. Metallo, T. L. Schneider, and A. Schepartz. 1999. DNA specificity enhanced by sequential binding of protein monomers. Proc Natl Acad Sci U S A 96:11735-11739. https://doi.org/10.1073/pnas.96.21.11735.

40. Sawers, R. G., E. Zehelein, and A. Bock. 1988. Two-dimensional gel electrophoretic analysis of Escherichia coli proteins: influence of various anaerobic growth conditions and the fnr gene product on cellular protein composition. Arch Microbiol 149:240-244.

41. Wang, L., L. Zhang, Z. Liu, D. Zhao, X. Liu, B. Zhang, J. Xie, Y. Hong, P. Li, S. Chen, R. 
Dixon, and J. Li. 2013. A minimal nitrogen fixation gene cluster from Paenibacillus sp. WLY78 enables expression of active nitrogenase in Escherichia coli. PLoS Genet 9:e1003865. https://doi.org/10.1371/journal.pgen.1003865.

42. Xie, J. B., L. Q. Bai, L. Y. Wang, and S. F. Chen. 2012. Phylogeny of 16S rRNA and nifH genes and regulation of nitrogenase activity by oxygen and ammonium in the genus Paenibacillus. Mikrobiologiia 81:760-767. https://doi.org/10.1134/S0026261712060173.

43. Gouet, P., X. Robert, and E. Courcelle. 2003. ESPript/ENDscript: Extracting and rendering sequence and 3D information from atomic structures of proteins. Nucleic Acids Res 31:3320-3323. https://doi.org/10.1093/nar/gkg556.

44. Kumar, S., G. Stecher, and K. Tamura. 2016. MEGA7: Molecular Evolutionary Genetics Analysis Version 7.0 for Bigger Datasets. Mol Biol Evol 33:1870-1874. https://doi.org/10.1093/molbev/msw054.

45. Anders, S., and W. Huber. 2010. Differential expression analysis for sequence count data. Genome Biol 11:R106. https://doi.org/10.1186/gb-2010-11-10-r106.

FIG 1 Homology analysis of Fnr proteins and phylogenetic analysis selected from Crp/Fnr superfamily in Firmicutes. (A) Alignments of Fnr proteins from P. polymyxa, B. cereus and $B$. subtilis. Conserved cysteines required for binding of the $[4 \mathrm{Fe}-4 \mathrm{~S}]^{2+}$ are indicated by red arrows. The double underlined sequence represents the region of the N-terminal DNA-binding domain. The red underlined sequence represents the region of sensory domain. Bs, B. subtilis; Bc, B.

FIG 2 The growth curve and nitrogenase activity of the $f n r$ deletion mutants. (A) Influence of the fnr deletion on growth under anaerobic condition. P. polymyxa WLY78 and the fnr deletion mutants were cultivated in nitrogen deficient medium with casamino acid and no oxygen. (B) activity of $P$. polymyxa WLY78 and the fnr deletion mutants was measured by acetylene reduction assay when grown anaerobically in nitrogen deficient medium. 
assays (EMSA). (A) Consensus sequence of the predicted Fnr-binding sites. (B) In vitro binding of $\mathrm{NHis}_{6}$-Fnr1 and Fnr3-CHis ${ }_{6}$ to promoter region of some Fnr target genes. 179-442 bp DNA with final concentration of 0.03 pmol was used. The '-' in Lane 1 indicates EMSAs without $\mathrm{NHis}_{6}{ }_{-}$-Fnr1 or Fnr3-CHis ${ }_{6}$. Lanes 2-5 contained increasing concentrations $(0.05$, 0.2, 2, 6 $\mu \mathrm{M})$ of $\mathrm{NHis}_{6}$-Fnr1 or Fnr3-CHis 6 as indicated by the height of the triangle above the gel. S and $\mathrm{N}$ indicate competition assays with a 100-fold excess of unlabelled specific probe and nonspecific competitor DNA, respectively. Arrowheads: free probes. Brackets: DNA-protein complexes.

FIG 4 Differential expression of the $n i f$ genes and iron transport genes. (A) Differential expression of the 9 nif genes. (B) Differential expression of the genes involved in iron transport. FC in $\log _{2} \mathrm{FC}$ indicates fold change (the read count ratio of $\Delta$ fnrl3 and wild type).

FIG 5 Differential expression of the genes related to anaerobic respiration and energy metabolism. (A) Schematic representation of the probable components of anaerobic respiration and energy metabolism in P. polymyxa WLY78 based on the genome annotation. Gray area represents the components whose transcripts are down-regulated in $\Delta f n r 13$. (B) Differential expression of the genes represented in the schema (A), FC in $\log _{2} \mathrm{FC}$ indicates fold change. The horizontal line above or down genes indicates these genes are in the same transcription unit. The asterisk indicates that the promoter region of the gene contains predictive Fnr-binding site.

FIG 6 Differential expression of the genes related to electron transport and carbon metabolism. (A) Differential expression of the genes in electron transport. (B) Differential expression of the genes in carbon metabolism. (C) Schematic representation of the probable components of carbon metabolism (glycolysis, TCA cycle and fermentation) in P. polymyxa WLY78 based on the genome annotation. Blue and red indicate the components whose transcripts are down-regulated and up-regulated in $\Delta f n r 13$, respectively. For abbreviation: P, phosphate; DHAP, dihydroxyacetone phosphate; PEP, phosphoenolpyruvate. The horizontal line above genes indicates these genes are in the same transcription unit. Asterisk indicates that the promoter region of the gene contains the predictive Fnr-binding sites. 
Fnr_BS $\quad \ldots \ldots \ldots$ MNFLSVRPSDSDLISSDLYELIESISTKRKME KHTYLFREGMDAEELYLIQSGLIEIGKLTSDGKDLTLRIC 72 FnY BC $\quad \ldots \ldots \ldots \ldots \ldots$ MANSMTLSQDLKELLASVEYKMQIKKGSFIFQEGMEATELYIIHSGKVQISKLSADGQELTLRIC 65 Fnr $\overline{1}$ Pp MQATCMERFGDEAERTGIQIYLTDEHFDLIKDIMSWKKVKAGVMLFREGDDAEQLYYIHSGHVKLRKSTEDGKELILTIQ 80 Fnr3_Pp $\quad \ldots \ldots \ldots \ldots \ldots \ldots \ldots \ldots \ldots \ldots \ldots \ldots \ldots \ldots \ldots \ldots \ldots$ MMKDMPESSHLFWEGDTADKLFYIKRGRVKVTKTTDEGKELILYMY 48 Fnr5_Pp ...MREIQDQEQLKYFLQLHELESILYEPLHPYLSLHRLE QGEKLCLQGDPIEHLYILVQGKVKIYTSSTEGKTLILCFK 77

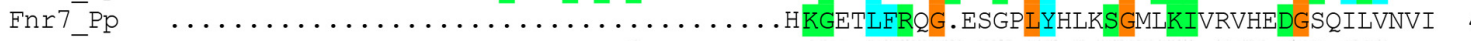
Consensus g lfregd ae $l_{Y} i \mathrm{sg}$ vki $\mathrm{k}$ t dgk lil

Fnr_BS QKHDIVGELTLFTEEPRYMLSÄKVLEDGEVLVINKNKLEKELIQNGÄLTFEFMKWMSTHLRKIQSKIRDL.LIHGKKGAL 151 Fnr BC SAYDIIGELTLFTDNAKYLLNSKCLEDVEVGVIKREALEKELLQKPALVFEFMKWI SEHLRRMOTKFRDL. VLHGKKGAL 144 Fnrī_Pp HQGDLIGEFSGIDGE. EYSCTAETADVCELGVVLVKDLES LLSKNGEMALQFIQWMAMKQRIMOSRFRDL. LLYGKTGAL 158 Fnr3 3 PP QSGDLVGQADPFFGT. KHSFSAEVLEDSDIGVIDHTDLELLICQHCDFAIDFMKWMGSHHRITQTKFRDL.MMYGKPGAL 126 Fnr5_PP TPIEVIGDVEYIRNS. . HVINTVEAVSPIYVIGIHHQWMNKYGKDYAPLLQFLLDVVTRKFCLDSDESSFNLMYPVEVRL 155 Fnr7_PP VPDEIIPHHSLISPN. PYYGTAVALVTSEVEVLSQKEWYQALEQDPTQCRAIALQLQDKLRMMQQRIDQL.TEVSPAEKL 118 Consensus

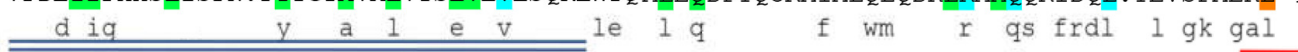

Fnr BS YSTLIRLSNSYGVERSDGILINIVLTNODLAKFCAAARESVNRMLGDIRKKKGVISIDESGKIILHKRDYLRCEIEC . .E 228 Fnr_BC Fnrī_Pp Fnr3 3 Pp Fnr5_Pp Enr $7_{-}^{-}$Pp YSTLIRMTNSYGILKENGILIDLPLTNQELANFCATSRES VNRMLNELKKOGTISIHK. GKITIHDLQFLKCEIAC. . E 220 ASTLIRASNTCGVAVKDGILLNMKLSHSELGEMIGATRESVNRMLSALKEQGTLDTRD. GKIVIHDLKVLRGMCCCPSYG 237 CSTLIRLCNTYGEPHGEHILIHKKITHTDLSNMIGATRESVNRMLSDLRKKKNAVEYDN. GMIVVKDIEMLQGVCHC . . E 202 ASYLLSVS . FDESNTAFHEELRASSLVDVANLIGTSYRHLNRVIRKMIEDGWVERTK. GYIVIRDRKGLS . . . . . . E 224 Consensus stlirlsnsyg il $\quad$ t d iga resvnrml $l g d \mathrm{~g}$ iv $\mathrm{d}$ ir $c$

$\begin{array}{lll} & \boldsymbol{1} & \\ \text { Fnr_Bs } & \text { NCPLEICNI } & 237 \\ \text { Fnr_BC } & \text { DCSASVCSI } & 229 \\ \text { Fnr1_Pp } & \text { OCAHEICRL } & 246 \\ \text { Fnr3_Pp } & \text { LCPREICRI } & 211 \\ \text { Fnr5_Pp } & \text { LAGHNIYES } & 233 \\ \text { Fnr7_Pp } & \text { NASR..... } & 175 \\ \text { Consensus } & \text { C eic } & \end{array}$

B

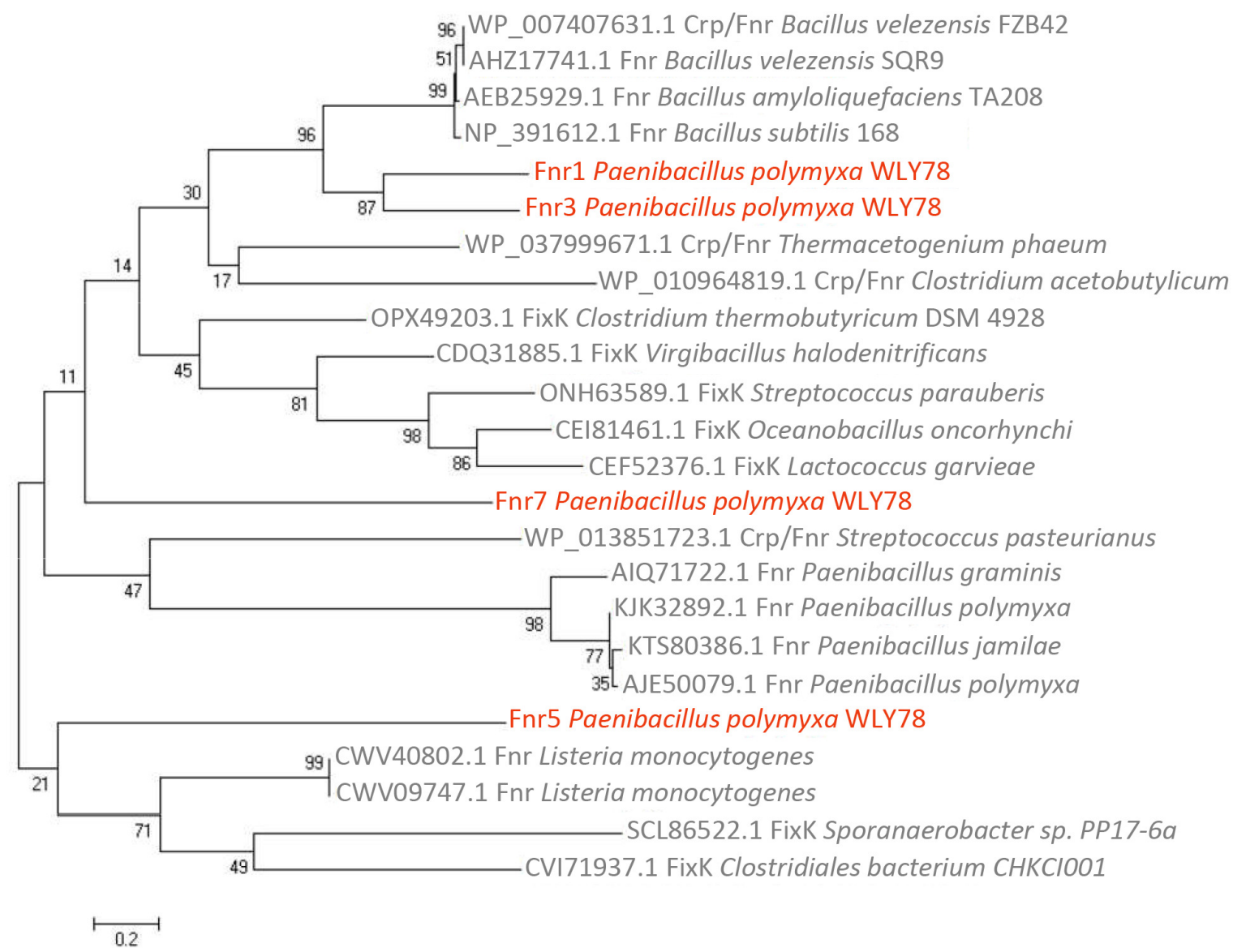



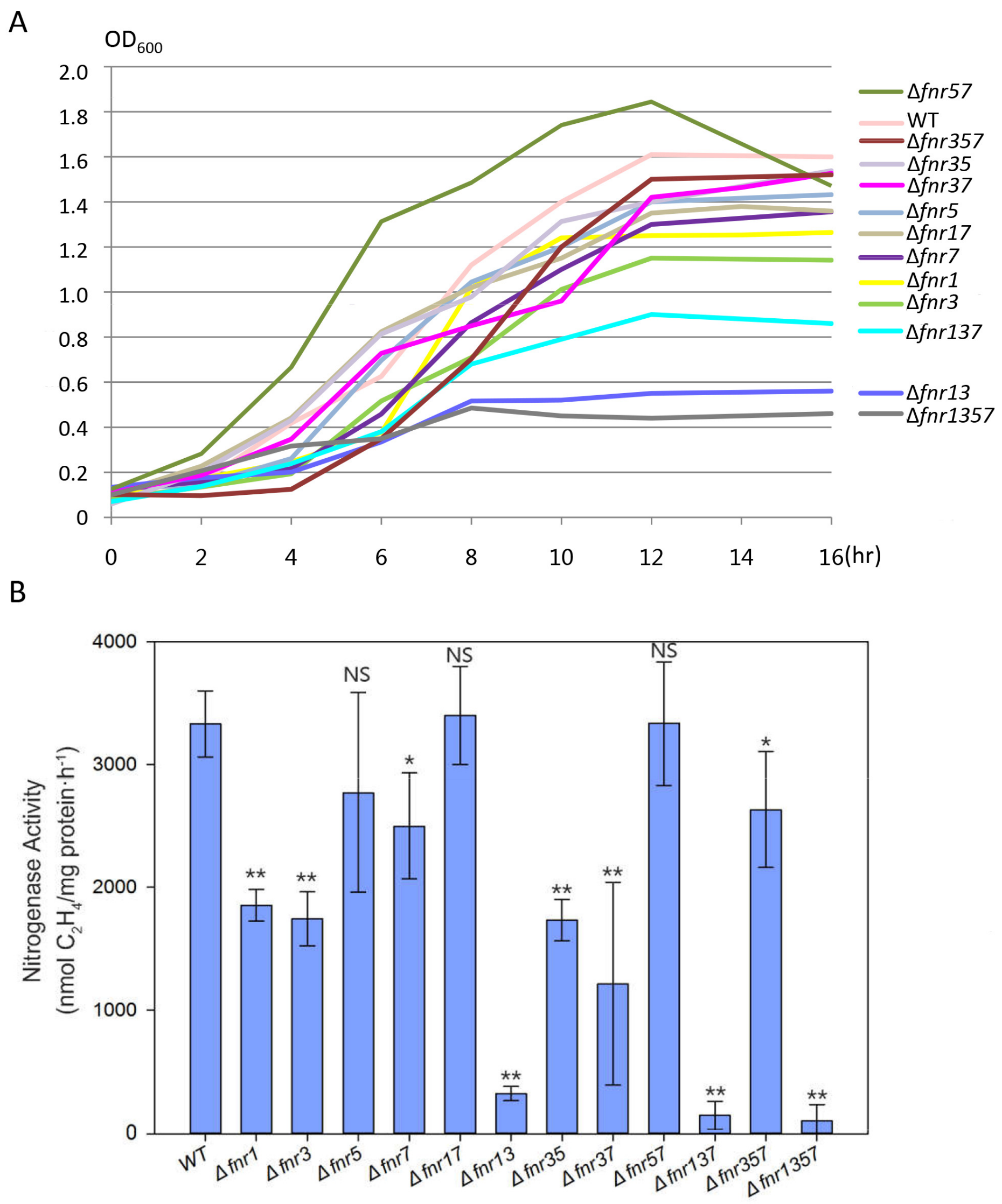
A

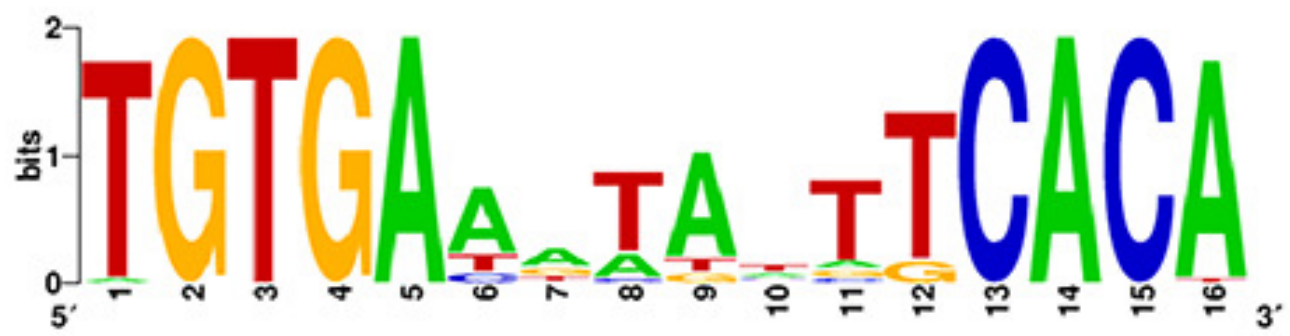

B

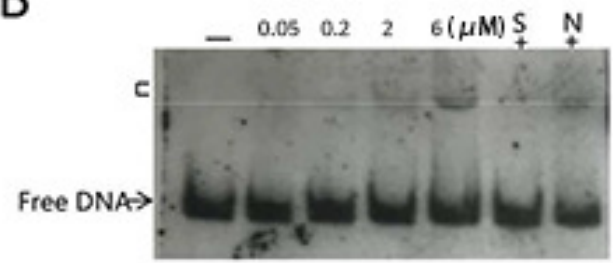

$\mathrm{Fnr} 1+\mathrm{PqO} \times A B C D$

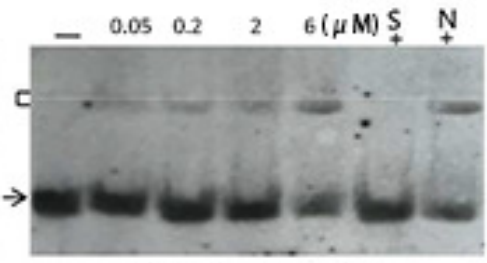

$\mathrm{Fnr} 3+\mathrm{PqO}$ XABCD

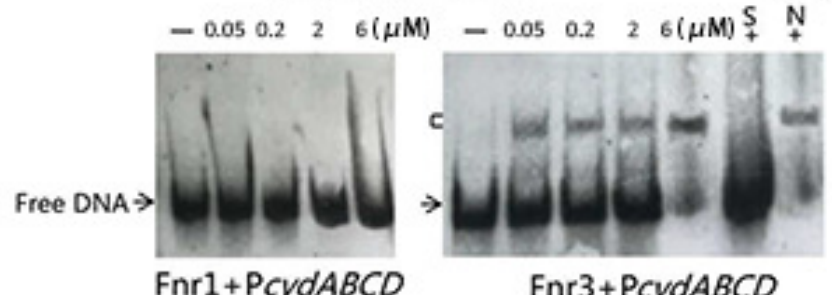

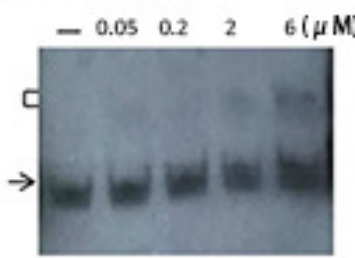

Fnr1+Pndh

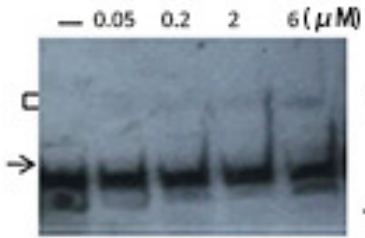

Fnr1+PhernN3

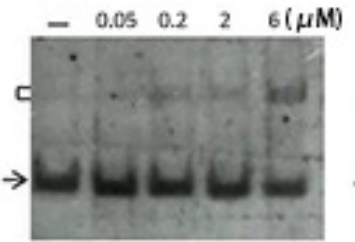

Fnr1+PpflBA

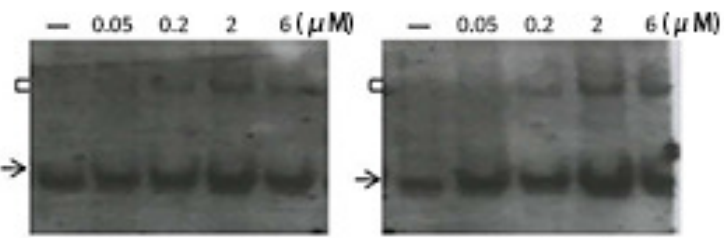

Fnr1+PnrdDG

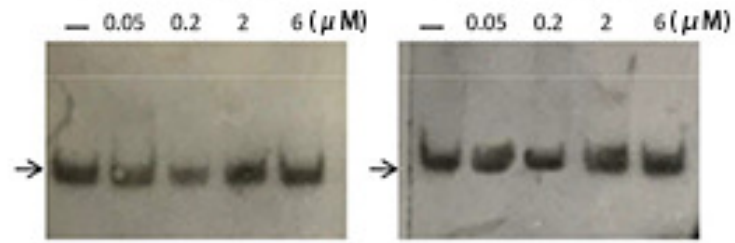

Fnr1+Pnifp

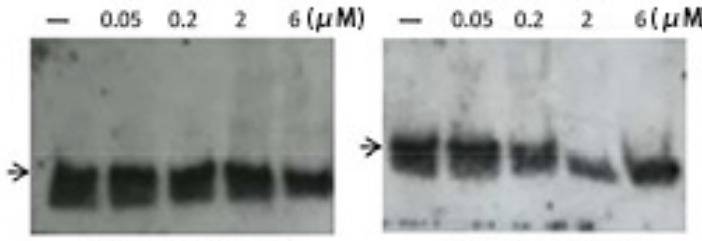

$\mathrm{Fnr} 1+\mathrm{P} g / n R A$

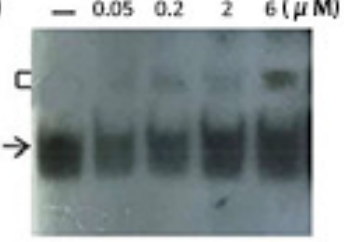

Fnr3+Pndh

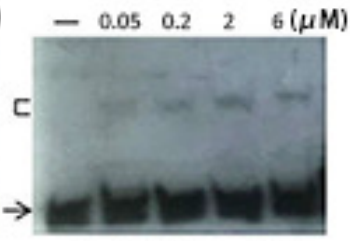

Fnr3+PhemN3

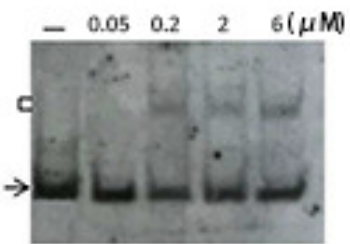

$\mathrm{Fnr} 3+\mathrm{P} p$ flBA

Fnr3+PnrdDG

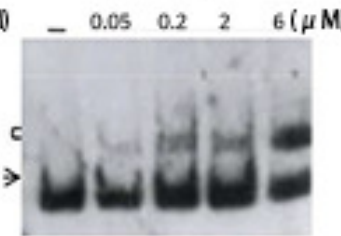

Fnr3 + PresDEp
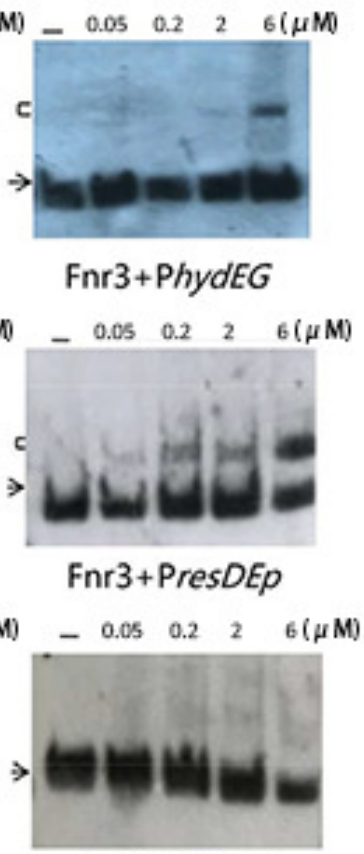

$\mathrm{Fnr} 3+\mathrm{PfeoAB}$
$\mathrm{Fnr} 1+\mathrm{PfeoAB}$

$\begin{array}{llll}-0.05 & 0.2 \quad 2 \quad 6(\mu \mathrm{M}) \\ \text { Free DNA> } & \end{array}$

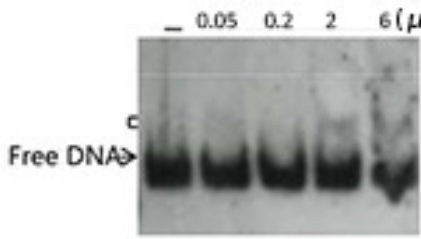




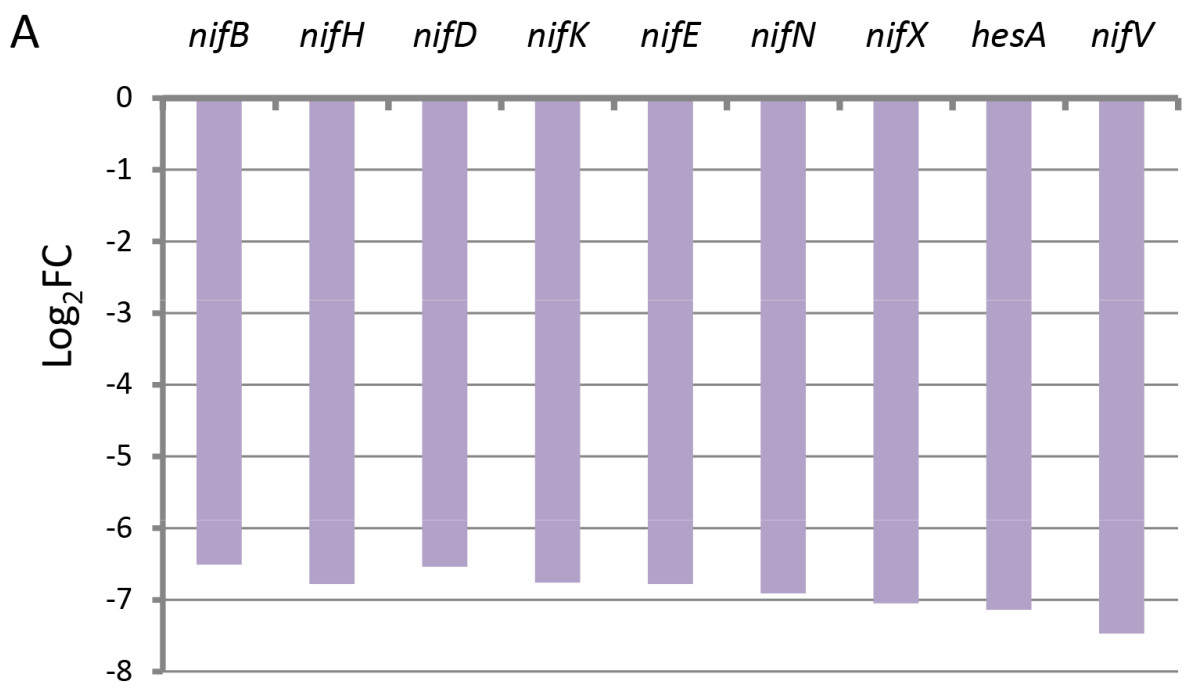

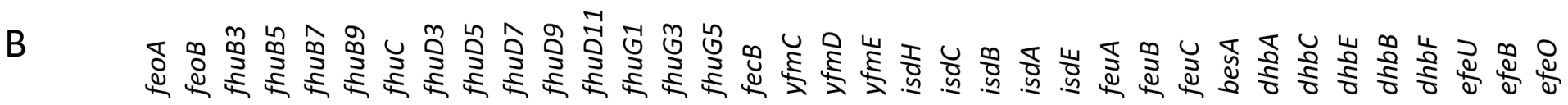

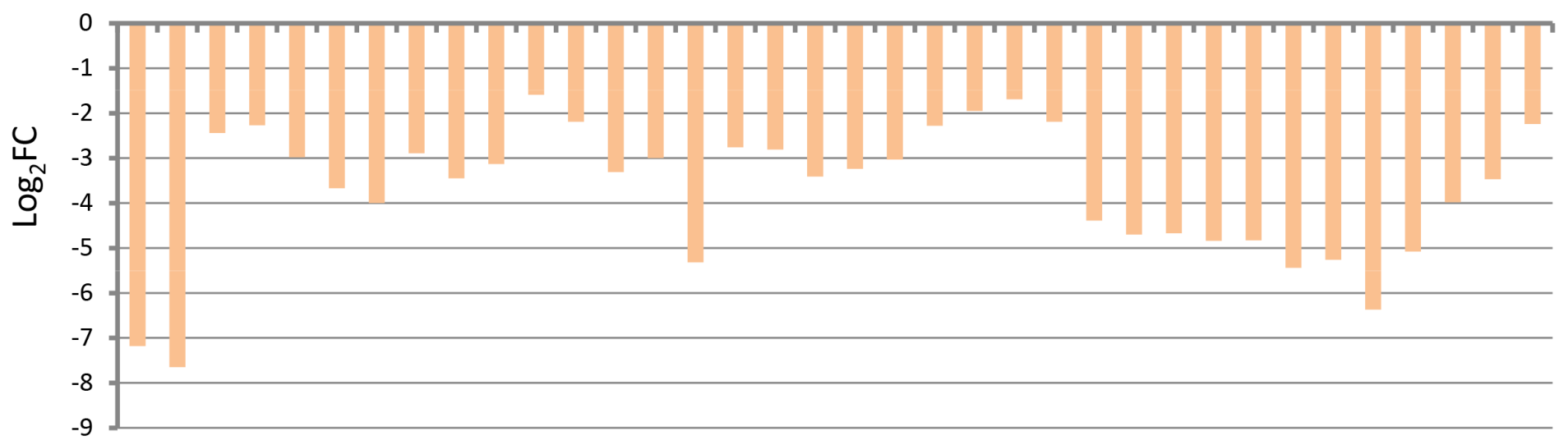




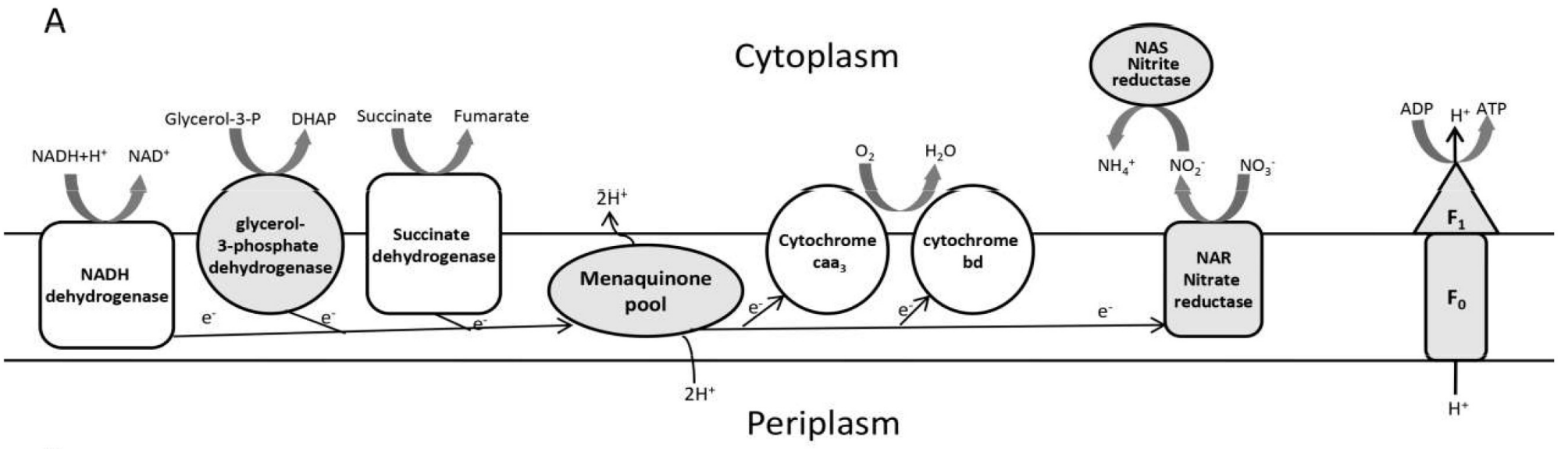

B

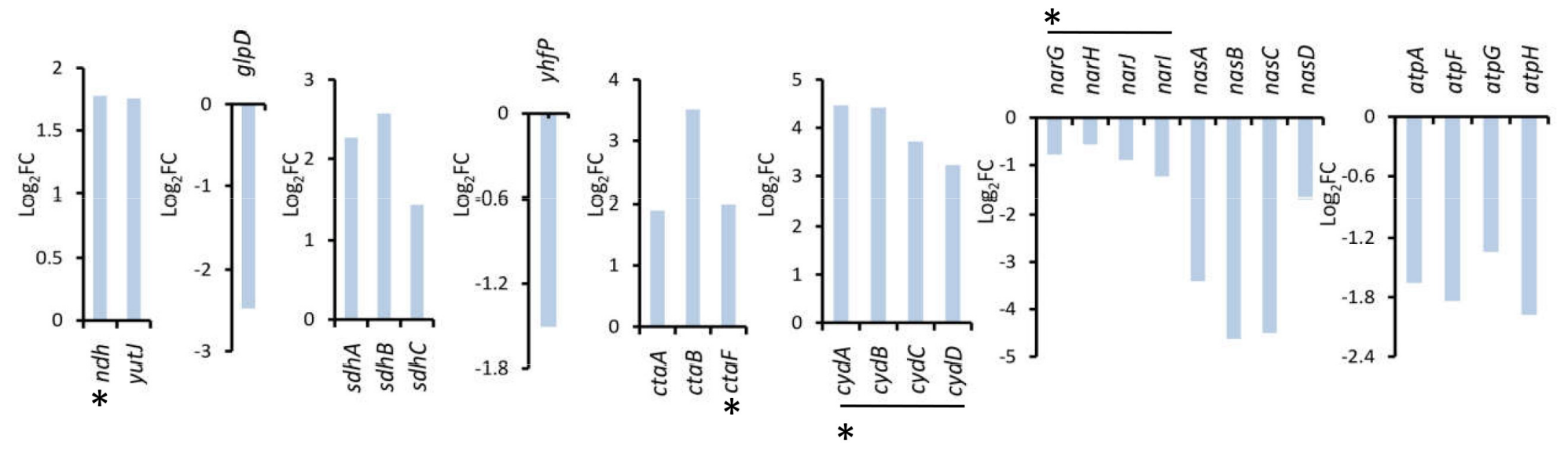



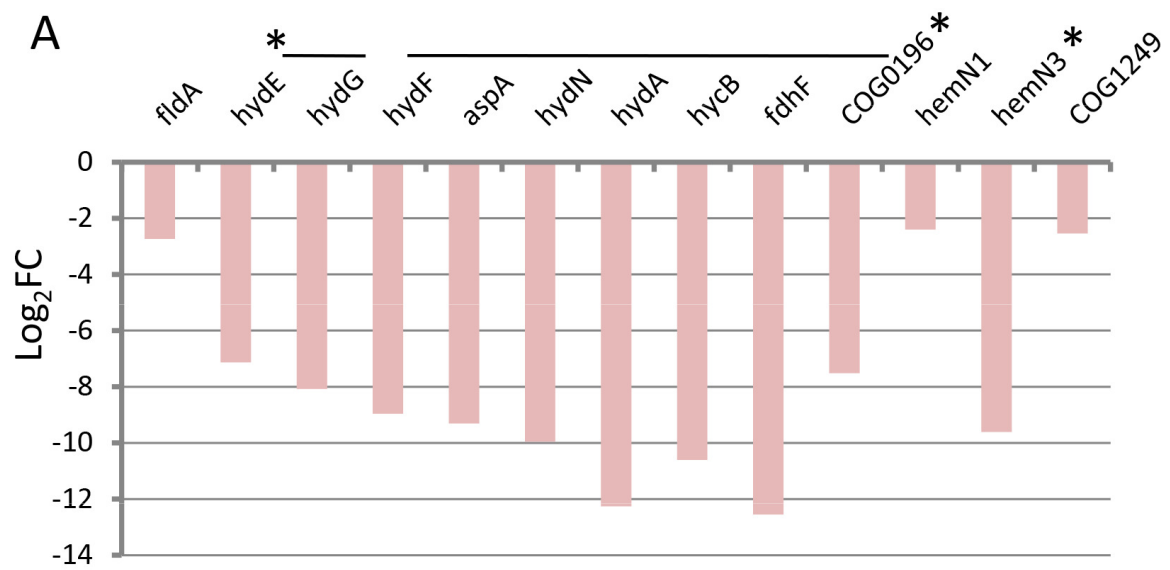

B

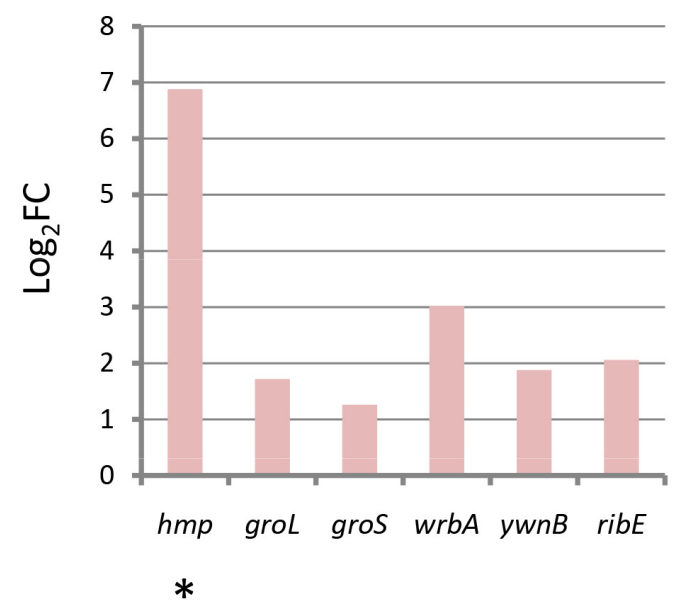

C Glucose

$\downarrow$ ptsG $\quad$ Glycolysis

Glucose-6-P

pgi

Fructose-6-P

$p f k A \mid \uparrow f b p$

DHAP $\underset{t p i}{\stackrel{\text { Gructose }}{\longrightarrow} \text { Glyceldehyde-3-P }}$

gapA $\mid \uparrow$ gapB

1,3-Bis-P-glycerate

$\uparrow p g k$

3-P-glycerate

$\uparrow p g m$

2-P-glycerate

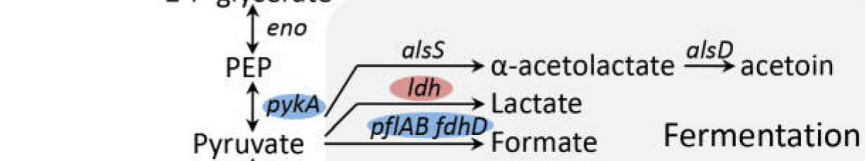

Pyruvate $\stackrel{p f \mid A B f d h D}{\longrightarrow}$ Formate pdhABCD

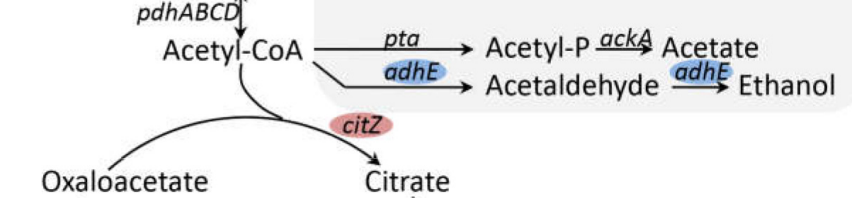

cith

Malate
citG $\uparrow$
Fumarate

TCA cycle

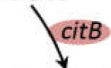

Isocitrate
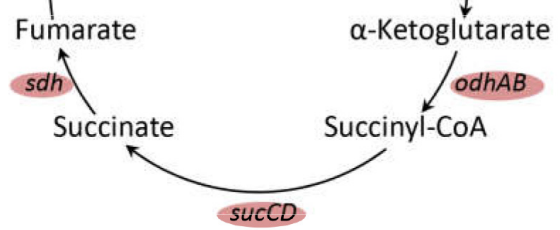
Table 1 The prediction of Fnr target genes in P. polymyxa WLY78

\begin{tabular}{|c|c|c|c|c|c|}
\hline \# & $\begin{array}{l}\text { Accession } \\
\text { number }\end{array}$ & Gene & Binding site & Function & E-value \\
\hline \multicolumn{6}{|c|}{ Carbon metabolism(11) } \\
\hline 1 & GM005029 & $y f k L$ & TGTGAAATGATTCAC & Fucose permease & 0.0032 \\
\hline 2 & GM001790 & nolL & TGTGAAAAAAATCAC & Fucose 4-O-acetylase or related acetyltransferase & 0.0081 \\
\hline 3 & GM000137 & COG3957 & TGTTCAAAATTTCACA & Probable phosphoketolase & 0.43 \\
\hline 4 & GM000073 & $c a h$ & $\begin{array}{l}\text { TGTACAATAATTTACA } \\
\text { TGTAAAAAGTTTCACA }\end{array}$ & Carbonic anhydrase & 2.7 \\
\hline 5 & GM003747 & $p g l$ & TGTGACGTTGTCCACA & 3-carboxymuconate cyclase & 4.2 \\
\hline 6 & GM003753 & $z w f 1$ & TGTGACATATTTAACA & Glucose-6-phosphate dehydrogenase & 4.3 \\
\hline 7 & GM002776 & $y v d A$ & CGTGAATCACATCAA & Putative carbonic anhydrase & 5.4 \\
\hline 8 & GM000674 & hxlA & AGTGAAGTTAATAAC & 3-hexulose-6-phosphate synthase & 6.6 \\
\hline 9 & GM000603 & gтис & TGTGAAATTGAGGAC & Phosphotransferase system cellobiose-specific component & 6.9 \\
\hline 10 & GM002477 & rpe & AAGGACATATTTCAC & Ribulose-phosphate 3-epimerase & 7.3 \\
\hline 11 & GM005208 & $\operatorname{agaR}$ & TCTGCATATTATCACA & Alpha-galactosidase & 9.4 \\
\hline \multicolumn{6}{|c|}{ Energy production and conversion(23) } \\
\hline 12 & GM003973 & yiaY & TGTGAAATATTTCACT & Alcohol dehydrogenase & 0.0046 \\
\hline 13 & GM000872 & nox & $\begin{array}{l}\text { TGTGAAAAATATCACA } \\
\text { AGTGAAATAAATCACA } \\
\text { CGTGAAACTATTCACA }\end{array}$ & NADH oxidase & 0.0052 \\
\hline 14 & GM002510 & $\operatorname{adh} E$ & $\begin{array}{l}\text { TGTGATTTAATTCACT } \\
\text { TGTGAAAAAAATCACA }\end{array}$ & Aldehyde alcohol dehydrogenase 2 & 0.0081 \\
\hline 15 & GM002511 & pflAB & $\begin{array}{l}\text { AGTGACAAAAATCACT } \\
\text { TGTGATTTTTGTCACA } \\
\text { CGTGAAAAAAATCACA }\end{array}$ & Formate acetyltransferase & 0.05 \\
\hline 16 & GM000607 & hmp & $\begin{array}{l}\text { TGTGCGTTTTGTCATT } \\
\text { AGTGACTAGAATCACA }\end{array}$ & Flavohemoprotein & 0.12 \\
\hline 17 & GM001678 & hemA & TGTGATATAATTAAC & Glutamyl-tRNA reductase & 0.32 \\
\hline 18 & GM001257 & $c y d A B C D$ & $\begin{array}{l}\text { TGACACGATGTTCACA } \\
\text { TGTGAATACTATCACA }\end{array}$ & Cytochrome bd ubiquinol oxidase subunit 1 & 0.36 \\
\hline 19 & GM002718 & COG0196 & TGTAATGAATTTCACA & FAD synthase & 0.38 \\
\hline 20 & GM001815 & COG4988 & CGTGATTTACATCACA & Cytochrome bd biosynthesis & 0.39 \\
\hline 21 & GM000687 & $n d h$ & $\begin{array}{l}\text { TATGATGTACCTCGCA } \\
\text { TGTGAAGCCTGTAACA }\end{array}$ & NADH dehydrogenase & 0.51 \\
\hline 22 & GM002710 & hydEG & TGTGAAACTAGTCAC & {$[\mathrm{FeFe}]$ hydrogenase maturase subunit } & 0.62 \\
\hline 23 & GM001268 & $q o x A B C D$ & AGAGATATTTTTCACA & Cytochrome bo(3) ubiquinol oxidase subunit 2 & 0.69 \\
\hline 24 & GM000550 & pyrKD & TGTGAAAATAGTCAC & Dihydroorotate dehydrogenase & 0.73 \\
\hline 25 & GM003546 & nemA & $\begin{array}{l}\text { AGGGACATTCCTCACA } \\
\text { AGTGAAATAAATCACA }\end{array}$ & NADH:flavin oxidoreductases & 0.91 \\
\hline 26 & GM003369 & $\operatorname{narK}$ & TGTGACGTTGTTCACC & Nitrate/nitrite transporter & 1.3 \\
\hline 27 & GM001655 & yugK & $\begin{array}{l}\text { TGAGCGTAAATTCACA } \\
\text { GATGAACAAATTCACA }\end{array}$ & NADH-dependent butanol dehydrogenase & 1.8 \\
\hline 28 & GM003365 & narGHJI & TGTGATTCTAGTCACA & Nitrate reductase large subunit & 1.9 \\
\hline 29 & GM004675 & groLS & AGGGAGGTTTATCAC & $60 \mathrm{kDa}$ chaperonin & 2 \\
\hline
\end{tabular}


30

GM001980 $a d h C \quad$ AGTGATTATATTCACA

31 GM000072 adhP TGTGAAACTTTTTACA

33

34

35

Transport and binding protein(16)

GM000873

GM005031

GM002779

GM004353

GM004015

GM004154

GM004112

GM003729

GM002284

GM003198

GM000624

GM005322 ydeG

GM002114

GM005382

GM000626

$y t w F$

$y r h G$

$y f k L$

$s s u B$

$k h t T$

$m d f A$

lys 9

$y f m R$

yjm $B$

оррC

nhaP2

bcrA

$y d e G$

pcaC
COG1277 TGCGATGTTAATCCCA

Regulatory function(19)

\begin{tabular}{|c|c|}
\hline 52 & GM002508 \\
\hline 53 & GM005030 \\
\hline 54 & GM003754 \\
\hline & GM005657 \\
\hline & GM005658 \\
\hline & GM005659 \\
\hline & GM001867 \\
\hline 9 & GM001981 \\
\hline & GM002775 \\
\hline & GM001140 \\
\hline & GM002515 \\
\hline & GM002506 \\
\hline & GM000642 \\
\hline & GM000406 \\
\hline & GM002608 \\
\hline
\end{tabular}

TGTGATTTTTTTCACA AGTGAATTAAATCACA

AGTGAAAATATTCAC

TGTTAAATATGTCACA

AGTGATATTTATCACA

TGTGATAAATATCACT

TGTGAGGATGTTCAC

TGTGAGTAACATCAT

TGTCACAAATTTTACA

TGTGAATATAATCACT

TATGAAAAAAAGCACA

TGTGAACGACCTGACT

TGTGCTCAATCTCCCA

TGTGCTGTATCGCACA AGAGATGTATTTCACT

AATGACAATCATCAC

$\mathrm{Fe}^{2+} / \mathrm{Zn}^{2+}$ uptake regulation proteins

HTH-type transcriptional regulator

TTTGATTAACTTCACT

TCTGGTAAACTTCACA

AraC family transcriptional regulator

Single-stranded DNA-binding protein

TTTGAGGCATTTCACA Transcriptional regulator
2.2

2.7

3

3.7

9.1

9.9

0.21

0.031

0.059

1.5

1.4

0.27

2.1

2.8

3.6

8.3

3.1

4.7

6.4

7.6

8.9

9.9

0.034

0.059

0.18

0.27

0.45

1.1

1.3

2.2

2.2

2.5

4.4

4.5

6.6

6.7

7.4 


\begin{tabular}{|c|c|c|c|c|c|}
\hline 67 & GM001082 & $r s m C$ & AGTGAAATAATTGAC & 16S rRNA methyltransferase & 7.5 \\
\hline 68 & GM003772 & & TGTGAAACCTTTTACT & DNA-binding protein & 8.2 \\
\hline 69 & GM002171 & tet $R$ & TGTGATCTTGTGAACA & TetR family transcriptional regulator & 8.9 \\
\hline 70 & GM003579 & $y k o G$ & AGTGAAATAAAGCAT & PhoB family transcriptional regulator & 9.1 \\
\hline \multicolumn{6}{|c|}{ Amino acid metabolism(6) } \\
\hline 71 & GM002422 & $\operatorname{aroA}$ & TGTGCAAAATGTCAC & 3-phosphoshikimate 1-carboxyvinyltransferase & 0.21 \\
\hline 72 & GM003694 & $\operatorname{tcyK}$ & TGTTAAATTAATCACA & L-cystine-binding protein & 0.86 \\
\hline 73 & GM003040 & $\operatorname{gln} A$ & AGTGAGTTATAGCAG & Glutamine synthetase & 3.2 \\
\hline 74 & GM003471 & metB & TTTGAAATTTTTCAGA & Cystathionine gamma-synthase & 6.2 \\
\hline 75 & GM004111 & $y h f E$ & TGTGATCTATGACACG & Putative aminopeptidase & 7.3 \\
\hline 76 & GM000557 & $y b g G$ & TGTGAAAAAAAGAAC & Homocysteine methyltransferase & 9.6 \\
\hline \multicolumn{6}{|c|}{ Nucleotide metabolism(3) } \\
\hline 77 & GM000911 & upp & TGTGATAAAAGTCAC & Uracil phosphoribosyltransferase & 0.11 \\
\hline 78 & GM000788 & $n r d D$ & TGTGACAATAGTCAC & Anaerobic ribonucleoside-triphosphate reductase & 0.73 \\
\hline 79 & GM001247 & uppP2 & TGTGAAATTTATCATT & UDP pyrophosphate phosphatase & 1.6 \\
\hline \multicolumn{6}{|c|}{ Lipid metabolism(2) } \\
\hline 80 & GM002581 & & ACTGAGTATATGCAC & O-antigen ligase family protein & 4.4 \\
\hline 81 & GM002539 & $a c c B$ & $\begin{array}{l}\text { TGTCCAATATGTTACA } \\
\text { TGTGATATAATACATA }\end{array}$ & Biotin carboxyl carrier protein of acetyl-CoA carboxylase & 5.7 \\
\hline
\end{tabular}

Replication, recombination and repair(5)

\begin{tabular}{|c|c|c|c|c|c|}
\hline 82 & GM002744 & $\operatorname{crfC}$ & TGTGATGATTCTCACA & Replication fork clamp-binding protein & 1.4 \\
\hline 83 & GM001022 & $u v r A$ & TTTGCGATATATCACT & UvrABC system protein & 2.6 \\
\hline 84 & GM002634 & hupA & TGTGCAATATTTCGCC & DNA-binding protein & 4 \\
\hline 85 & GM003041 & $x e r C$ & TGTGAAGTAGTGGTC & Tyrosine recombinase XerC-like & 4.5 \\
\hline 86 & GM000535 & yurQ & TGTGATAATATTTACA & Nuclease subunit of the excinuclease complex & 6.6 \\
\hline \multicolumn{6}{|c|}{ Coenzyme transport and metabolism(6) } \\
\hline 87 & GM001455 & $m q n E$ & TGTAACATTTTTCACA & Aminodeoxy futalosine synthase & 0.29 \\
\hline 88 & GM001456 & sufA & TGTGAAAAATGTTAC & Iron-sulfur cluster assembly protein & 0.29 \\
\hline 89 & GM004949 & hemN3 & TGTGATCTATTTCATA & Coproporphyrinogen III oxidase & 0.58 \\
\hline 90 & GM001229 & $p d x K$ & TGTGACTTTTAGCATA & Pyridoxine kinase & 1.7 \\
\hline 91 & GM001483 & $\operatorname{trx} A$ & TGTGATATAATTAAA & Thioredoxin & 7.8 \\
\hline 92 & GM002596 & $r i b E$ & TGTGACTCTCATCACC & Riboflavin synthase & 8.2 \\
\hline \multicolumn{6}{|c|}{ Cell wall/membrane/envelope biogenesis(15) } \\
\hline 93 & GM003974 & $n l p C$ & AGTGAAATATTTCAC & Cell wall-associated hydrolases & 0.032 \\
\hline 94 & GM005089 & yphA & $\begin{array}{l}\text { TGTGATTTTTATCACA } \\
\text { TGAGAACAAAGTCGCA }\end{array}$ & DoxX family membrane protein & 0.034 \\
\hline 95 & GM001894 & ulaA & TGTGATTTAATTCACA & Membrane protein & 0.15 \\
\hline 96 & GM000012 & $y o z B$ & TGTGAAATTGTTCATA & Uncharacterized membrane protein & 0.34 \\
\hline 97 & GM003888 & cheW & TGTGAAGTTGCTGAC & Chemotaxis protein & 0.86 \\
\hline 98 & GM004239 & yrkA & TGTGAATATGTTCACG & Membrane protein & 0.9 \\
\hline 99 & GM002294 & murF & AGTGATGAAAAGAAC & UDP-N-acetylmuramoyl-tripeptide ligase & 3.2 \\
\hline 100 & GM002164 & $y d c F$ & TATGAAAAAAATCAC & Membrane protein & 5.5 \\
\hline 101 & GM001042 & $r f b D$ & TGTGCATATCGTCAAA & dTDP-4-dehydrorhamnose reductase & 5.7 \\
\hline
\end{tabular}




$\begin{array}{llllll}102 & \text { GM004014 } & y g a E & \text { TGTGACGTGTTGCAA } & \text { Aromatic acid exporter family protein } & 6.5 \\ 103 & \text { GM001502 } & c h e X & \text { TAGGACATTATTCACA } & \text { CheY-P phosphatase CheX } & 7.2 \\ 104 & \text { GM003651 } & y c g R & \text { AGGGAGGTGATTCAC } & \text { Membrane protein } & 7.6 \\ 105 & \text { GM002165 } & y v b V & \text { AGTGATTTTTTTCATA } & \text { Membrane protein } & 9.1 \\ 106 & \text { GM004741 } & g a l U 2 & \text { AGTGTTATACATCACT } & \text { Polysaccharide polymerase } & 9.3 \\ 107 & \text { GM003112 } & s p r & \text { AGCAAAATATTGCAC } & \text { Lipoprotein } & 9.5\end{array}$

Cell cycle control, cell division(1)

108 GM001289 cyk3 TGTGATAAAATTCGCT $\quad$ Uncharacterized protein involved in cytokinesis $\quad 6.4$

Function unknown of unclassified proteins(36)

\begin{tabular}{|c|c|c|c|c|c|}
\hline 109 & GM003182 & & TGTGAAAAAAATCAC & Hypothetical protein & 0.008 \\
\hline 110 & GM001791 & & TGTGATTTTTTTCACA & Alpha/beta hydrolase & 0.19 \\
\hline 111 & GM002231 & & TGTGATACAGGTCAC & Hypothetical protein & 0.3 \\
\hline 112 & GM004946 & & AATGATGTATTTCACA & Hypothetical protein & 0.33 \\
\hline 113 & GM002092 & & TTTGCATAACATCACA & Hypothetical protein & 0.63 \\
\hline 114 & GM005088 & & TGTGATAAAAATCAC & Hypothetical protein & 0.83 \\
\hline 115 & GM003667 & $p d p B$ & TGTTATGAGGTTCACA & Hypothetical protein & 0.85 \\
\hline 116 & GM002873 & & TGTGCGGTATATCACT & Hypothetical protein & 0.86 \\
\hline 117 & GM003650 & $y c i C$ & TGTGAATCACCTCCCT & Putative metal chaperone & 1.1 \\
\hline 118 & GM000257 & COG4506 & TGTGAAGAATTGCAT & Hypothetical protein & 1.8 \\
\hline 119 & GM001872 & & AGTGAATCGTTTCACA & Hypothetical protein & 1.9 \\
\hline 120 & GM005310 & $y n d D$ & TGTGACTAGAATCAC & Spore gernimation protein & 1.9 \\
\hline 121 & GM000205 & & AGTGATTATTTACACA & Hypothetical protein & 2 \\
\hline 122 & GM005405 & $\operatorname{luxS}$ & $\begin{array}{l}\text { TGTACACTATGTCAAA } \\
\text { TTTGAAAAAAATCACA }\end{array}$ & S-ribosylhomocysteinase & 2.1 \\
\hline 123 & GM002194 & comEA & TGTAAAAATGCTCAC & Competence protein ComEA & 2.6 \\
\hline 124 & GM000606 & & $\begin{array}{l}\text { TGTGATTCTAGTCACT } \\
\text { AATGACAAAACGCACA }\end{array}$ & Coagulation factor type domain protein & 2.8 \\
\hline 125 & GM005406 & $y w a C$ & $\begin{array}{l}\text { TGTGATTTTTTTCAAA } \\
\text { TTTGACATAGTGTACA }\end{array}$ & GTP pyrophosphokinase & 3.6 \\
\hline 126 & GM000597 & lolD & TTCGAAATATTTCACA & Lipoprotein releasing system ATP-binding protein & 3.9 \\
\hline 127 & GM002803 & & TGTGATGTATCGCAA & Hypothetical protein & 4 \\
\hline 128 & GM004268 & & TGTGAGTGATTTCACT & Hypothetical protein & 5 \\
\hline 129 & GM004035 & & GTTGACGTGTTGCACA & Hypothetical protein & 5.1 \\
\hline 130 & GM000635 & & TGTGATTTACATCAAT & Hypothetical protein & 6.1 \\
\hline 131 & GM002233 & hit & TGGGAAATACATAAC & Hit family hydrolase & 6.1 \\
\hline 132 & GM005622 & yjiN & TGTGATTTAGTTCACG & GTP pyrophosphokinase & 6.5 \\
\hline 133 & GM000641 & xylA & AGTGAAGTTAATCAA & Beta-xylosidase & 6.6 \\
\hline 134 & GM001393 & & AGTGATGAAATGCCC & Hypothetical protein & 6.9 \\
\hline 135 & GM005304 & $m d p B$ & TATGACTAATGTCACA & Microcystin-dependent protein & 6.9 \\
\hline 136 & GM005298 & & CGTGATTTACATTACA & Hypothetical protein & 8.6 \\
\hline 137 & GM001951 & & TGTTCATAATATCACT & Cell wall assembly protein & 8.9 \\
\hline 138 & GM001573 & $y h c N$ & $\begin{array}{l}\text { AGTGATCTTTTTCAAA } \\
\text { TGATAATCTCGTCACA }\end{array}$ & Lipoprotein & 9.1 \\
\hline 139 & GM002844 & & TGTGCAGTTGATTACA & Hypothetical protein & 9.1 \\
\hline 140 & GM000664 & COG1917 & TGTGATTGCAATCACA & Cupin & 9.5 \\
\hline
\end{tabular}


141 GM003988

TGTGATTTTTTCCACT Hypothetical protein

9.6

142 GM001572

$\operatorname{lipA}$

TGTGACGAGATTATC

Alpha/beta hydrolase

9.9

143 GM005369

TGTGATTGTACGCACA Hypothetical protein 